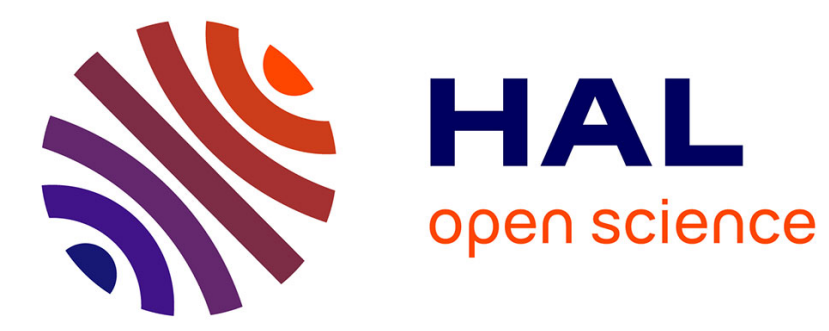

\title{
Quantum and Quantum-Classical Studies of the Photoisomerization of a Retinal Chromophore Model
}

\author{
Emanuele Marsili, Massimo Olivucci, D. Lauvergnat, Federica Agostini
}

\section{To cite this version:}

Emanuele Marsili, Massimo Olivucci, D. Lauvergnat, Federica Agostini. Quantum and QuantumClassical Studies of the Photoisomerization of a Retinal Chromophore Model. Journal of Chemical Theory and Computation, 2020, 16 (10), pp.6032-6048. 10.1021/acs.jctc.0c00679 . hal-02994954

\section{HAL Id: hal-02994954 https://hal.science/hal-02994954}

Submitted on 10 Nov 2020

HAL is a multi-disciplinary open access archive for the deposit and dissemination of scientific research documents, whether they are published or not. The documents may come from teaching and research institutions in France or abroad, or from public or private research centers.
L'archive ouverte pluridisciplinaire HAL, est destinée au dépôt et à la diffusion de documents scientifiques de niveau recherche, publiés ou non, émanant des établissements d'enseignement et de recherche français ou étrangers, des laboratoires publics ou privés. 


\section{Quantum and quantum-classical studies of}

\section{the photoisomerization of a retinal chromophore model}

Emanuele Marsili, ${ }^{\dagger}$ Massimo Olivucci, ${ }^{\ddagger}, \S$ David Lauvergnat, ${ }^{*} \dagger$ and Federica Agostini*, ${ }^{\dagger}$

$\dagger$ Université Paris-Saclay, CNRS, Institut de Chimie Physique UMR8000, 91405, Orsay, France

$\ddagger$ Department of Biotechnology, Chemistry and Pharmacy, Università degli Studi di Siena, Via A. Moro 2, I-53100 Siena, Italy

IDepartment of Chemistry, Durham University, South Road, Durham DH1 3LE, United Kingdom

$\S$ Department of Chemistry, Bowling Green State University, Bowling Green, Ohio 43403, United States of America

E-mail: david.lauvergnat@universite-paris-saclay.fr; federica.agostini@universite-paris-saclay.fr 


\begin{abstract}
We report an in-depth analysis of the photo-induced isomerization of the 2-cispenta-2,4-dieniminium cation: a minimal model of the 11-cis retinal protonated Schiff base chromophore of the dim-light photoreceptor rhodopsin. Based on recently-developed three-dimensional potentials parametrized on ab initio multi-state multi-configurational second-order perturbation theory data, we perform quantum-dynamical studies. In addition, simulations based on various quantum-classical methods, among which Tully surface hopping and the coupled-trajectory approach derived from the exact factorization, allow us to validate their performance against vibronic-wavepacket propagation and, therefore, a purely quantum treatment. Quantum-dynamics results uncover qualitative differences with respect to the two-dimensional Hahn-Stock potentials, widely-used as model potentials for the isomerization of the same chromophore, due to the increased dimensionality and three-mode correlation. Quantum-classical simulations show, instead, that three-dimensional model potentials are capable of capturing a number of features revealed by atomistic simulations and experimental observations. In particular, a recently reported vibrational phase relationship between double-bond torsion and hydrogen-out-of-plane modes critical for rhodopsin isomerization efficiency is correctly reproduced.
\end{abstract}

\title{
1. Introduction
}

The 11-cis retinal protonated Schiff base (rPSB11) is the chromophore of Rhodopsin (Rh), an animal opsin found in the vertebrate retina. ${ }^{10}$ In the Rh cavity, the photo-induced doublebond isomerization of rPSB11 occurs in less than 200 fs: ${ }^{[2}$ A process that, ultimately, leads to the stimulation of the optical nerve and vision. Due to its ultrafast speed and photobiological role, the rPSB11 isomerization has been studied for many years experimentally ${ }^{3-7}$ and computationally. $\frac{517-12}{-12}$ Atomistic simulations of the light-induced reaction dynamics of rPSB11 in Rh using ab initio electronic structure calculations ${ }^{13}$ have been accessible since 
the early 2000s accounting for the effect of the protein environment via hybrid quantummechanics/molecular-mechanics (QM/MM) methods.

When combined with dynamics studies based on classical trajectories, these studies have provided information on both the geometrical and electronic structure time evolution ${ }^{[5|10| 17}$ of the reaction. However, quantum-classical approaches usually employed in atomistic simulations propagate trajectories independently, and purely quantum effects, which would couple the trajectories, are thus neglected. When such effects are of interest, quantum-dynamical studies of low-dimensionality models based on parametrized potential energy surfaces (PESs) have the potential to provide complementary information ${ }^{21}$ (see also the Supporting Information of Ref. $\left.\frac{17}{}\right)$. In this context, it is interesting to investigate the case of the 2-cispenta-2,4-dieniminium cation (cis-PSB3). PSB3 is a minimal model of rPSB11 which retains certain basic features of the isomerization mechanism documented for the full rPSB11 chromophore even when this is embedded in the Rh cavity. Therefore, PSB3 has been employed for dynamics studies of the cis to trans photoisomerization in isolated conditions. $\stackrel{23 \mid 28}{37}$ Moreover, it is sufficiently small to allow for ab initio (wavefunction-based) multi-state multiconfigurational (MSMC) mapping of the electronic structure of ground $\left(S_{0}\right)$ and first singlet excited $\left(S_{1}\right)$ states, and for the development of reduced-dimensionality analytical models of PESs that can be used for quantum-dynamics studies. Here, by "reduced dimensionality" we mean the three modes $\frac{810[26 \mid 34] 36}{38}$ that drive the cis-trans isomerization of rPSB11 after light absorption. The modes are the bond-length-alternation (BLA) stretching, the torsional deformation around the reactive double bond (Tors), and the hydrogen-out-of-plane (HOOP) wagging. The same modes drive the photoisomerization of cis-PSB3, $\frac{3637}{37}$ confirming that this model is particularly suitable for qualitative studies.

In this work, we study the dynamics underlying the ultrafast isomerization of rPSB11like systems triggered by vertical $S_{0} \rightarrow S_{1}$ photo-excitation, employing the recently developed three-dimensional PESs (hereafter also called "three-dimensional model") of gas-phase PSB3, 27 based on XMCQDPT2 $2^{39}$ ab initio MSMC electronic-structure calculations. Our 
aim is to investigate the ability of the reduced-dimensionality model potentials to capture, at least qualitatively, critical features of the dynamics of Rh, such as its early decay time, ${ }^{6}$ vibrational coherence ${ }^{\sqrt{60}}$ and the probability of formation of the trans photoproduct. ${ }^{7}$ The proposed analysis is based on the propagation of vibronic wavepackets using ELVIBRoT ${ }^{41}$ and on four trajectory-based quantum-classical approaches to excited-state dy-

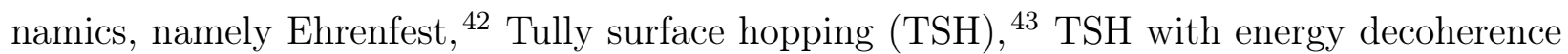
corrections (TSH-EDC), $\underline{44}$ and the coupled-trajectory mixed quantum-classical (CT-MQC) $\operatorname{approach}^{45}$ based on the exact factorization of the electron-nuclear wavefunction. .51152 On the one hand, quantum-dynamics results for the three-dimensional model ${ }^{27}$ are compared to the widely used $\frac{22|25| 38 \mid 53}{55}$ two-dimensional model proposed by Hahn and Stock $\frac{\sqrt[56]{5}}{\text { (here- }}$ after also called "two-dimensional model"), in order to highlight the crucial role played by the additional HOOP mode in driving the cis-trans isomerization. The study of the threedimensional model ${ }^{27}$ based on various quantum-classical schemes, on the other hand, is essential to put our analysis in perspective to atomistic simulations in full dimensionality. Therefore, it is clear that such an in-depth analysis and validation of the model would not be possible without the complementarity of information obtained based on quantum and quantum-classical dynamics. Furthermore, since our analysis relies on a common "force field" and on the benchmark provided by quantum-dynamics simulations, the findings presented in this work provide novel insight into different quantum-classical schemes, and in particular into the recently-developed CT-MQC approach, which is an analysis seldom reported in the literature. For all these reasons, our study goes beyond previous reports based on two-dimensional and/or empirically determined PES models. 21 23|25|38|53|56|57

The analysis of the results focuses on three main aspects of PSB3 captured dynamics: (i) the short-time energy distribution among the modes, (ii) the isomerization quantum efficiency, and (iii) the phase relationship between Tors and HOOP velocities necessary to achieve the trans product. With regard to point (i), we show that accounting for the quantum-mechanical character of nuclear dynamics appears to be surprisingly important 
for predicting energy redistribution and $S_{1}$-to- $S_{0}$ population transfer at short times after photo-excitation. Concerning point (ii), the analysis reveals the role of coherent vibrational motion in the excited state to enhance the funneling process through the $S_{1} / S_{0}$ conical intersection (CI) points ${ }^{3617110138}$ (or intersection space ${ }^{\sqrt{37}}$ ) and to determine the isomerization quantum efficiency. Finally, while points (i) and (ii) provide information useful to validate approximate methods, point (iii) examines the quality of the three-dimensional model for $\mathrm{Rh}$ isomerization and confirms the importance of phase matching between Tors and HOOP motion to access the trans stereochemistry.

Focusing on the technical aspects discussed in this work, the main points are: (iv) the development of the kinetic energy operator suitable for quantum-dynamics calculations in the curvilinear coordinates BLA, Tors, and HOOP, and (v) the proposition of suitable analysis strategies of CT-MQC trajectories, which appears to be necessary since the method lacks, in general, the intuitive picture in terms of hopping trajectories provided by the widely-used $\operatorname{TSH}(-\mathrm{EDC})$.

The paper is organized as follows. Sections 2 and 3 are devoted to the validation of approximate (quantum-classical) methods, and Section 4 examines the three-dimensional model from the application viewpoint. Accordingly, in Section 2, we describe the procedure to perform quantum dynamics simulations by defining the kinetic energy operator associated to the BLA, Tors and HOOP modes. In addition, we compare our quantum-dynamics results with the Hahn-Stock model. In Section 3, we investigate the performance of the quantumclassical schemes based on the comparison with quantum results for our reference threedimensional model PESs. In Section 4, we present an analysis purely based on trajectories to highlight the capability of the same model PESs to capture dynamical features observed in rPSB11-like systems from atomistic simulations. Thus, again, in Section 3 our PESs are consistently employed to validate quantum-classical schemes, while in Section 4 we explore their chemical significance. Section 5 summarizes our observations and discusses future developments. 


\section{Analysis of the model based on quantum dynamics simulations}

In this section we analyze the quantum vibronic wavepacket dynamics driven by the twostate three-dimensional model PESs developed in Ref. ${ }^{27}$ Note that the parametrization of the PESs has been slightly modified with respect to Ref., 27 and we describe the new potentials in Appendix A. In the following we describe:

- the curvilinear coordinates that define the three-dimensional model;

- the kinetic energy operator and the metric tensor associated to those curvilinear coordinates.

The crucial step before proceeding with the analysis of the dynamics is the derivation of the kinetic energy operator associated to the curvilinear coordinates employed in the model: BLA, Tors and HOOP, schematically represented in Fig. 1. The BLA stretching is defined

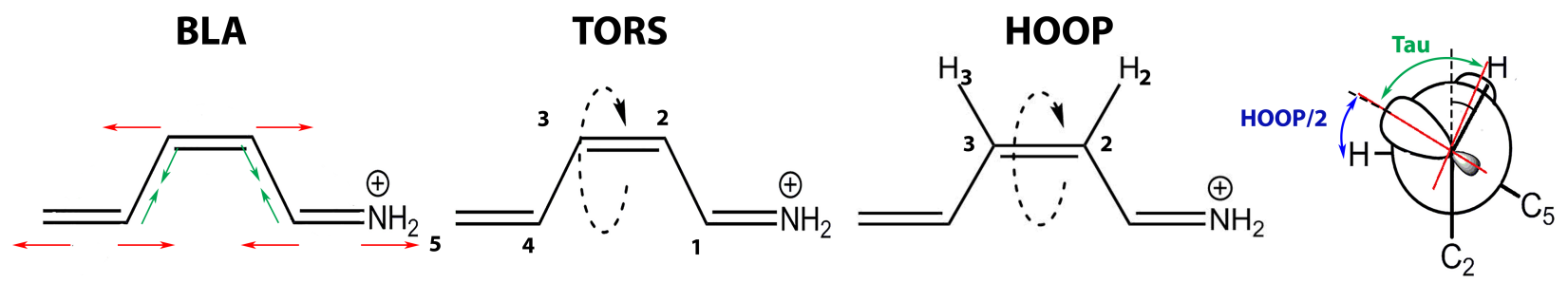

Figure 1: Schematic representation of the vibrational modes BLA, Tors, and HOOP. The relation between HOOP and an additional coordinate, Tau, is shown on the right: Tau = Tors $-\mathrm{HOOP} / 2$ will be used for the analysis presented below.

as the difference between the average length of the single bonds and the average length of the double bonds in the ground-state PSB3 Lewis formula, 1

$$
r=\frac{d_{C_{1} C_{2}}+d_{C_{3} C_{4}}}{2}-\frac{d_{N C_{1}}+d_{C_{2} C_{3}}+d_{C_{4} C_{5}}}{3}
$$

\footnotetext{
${ }^{1}$ Note that in the reference cis ground-state conformation, PSB3 has two single bonds, i.e., $\mathrm{C}_{1} \mathrm{C}_{2}$ and $\mathrm{C}_{3} \mathrm{C}_{4}$, and three double bonds, i.e., $\mathrm{NC}_{1}, \mathrm{C}_{2} \mathrm{C}_{3}$ and $\mathrm{C}_{4} \mathrm{C}_{5}$.
} 
where $d_{C_{A} C_{B}}$ is the distance between the atoms $C_{A}$ and $C_{B}$, using the indices of the carbon atoms given in Fig. 1 (similarly, $d_{N C_{1}}$ is the distance between the nitrogen and the carbon atom labelled 1); Tors identifies the torsional deformation around the reactive $\mathrm{C}_{2}=\mathrm{C}_{3}$ double bond, thus it is the dihedral angle formed by the carbon atoms $\mathrm{C}_{1} \mathrm{C}_{2} \mathrm{C}_{3} \mathrm{C}_{4}$,

$$
\theta=\operatorname{dihedral}\left(\mathrm{C}_{1} \mathrm{C}_{2} \mathrm{C}_{3} \mathrm{C}_{4}\right)
$$

the HOOP wagging mode is described by the difference between Tors and the $\mathrm{H}_{2} \mathrm{C}_{2} \mathrm{C}_{3} \mathrm{H}_{3}$ dihedral angle,

$$
\phi=\text { dihedral }\left(\mathrm{C}_{1} \mathrm{C}_{2} \mathrm{C}_{3} \mathrm{C}_{4}\right)-\text { dihedral }\left(\mathrm{H}_{2} \mathrm{C}_{2} \mathrm{C}_{3} \mathrm{H}_{3}\right) .
$$

The coordinates BLA, Tors and HOOP are used to calculate numerically the positiondependent metric tensor following the TNUM scheme. ${ }^{58}$ First, all 42 coordinates (including the center of mass degrees of freedom) belonging to PSB3 must be defined via the following coordinates transformations: (i) transformation to/from Z-matrix coordinates, (ii) linear combinations of internal coordinates, (iii) active transformation. Transformation (i) allows us to define the Z-matrix, relating Cartesian coordinates to the internal coordinates used in the quantum chemistry calculations for the parametrization of the potentials. ${ }^{27}$ Transformation (ii) is used to relate the Z-matrix coordinates to the active coordinates BLA, Tors and HOOP, such that they can be expressed as linear combinations of internal coordinates, as given in Eqs. (1), (2) and (3). The remaining linear combinations are generated automatically by TNUM. $\frac{58}{5}$ Transformation (iii) enables to set up the constraints to get the reduced-dimensionality model, and thus to select the three active coordinates. In the present study, rigid contraints (values at the cis equilibrium geometry) are defined on all coordinates except for the three active ones, i.e., Tors, BLA, and HOOP.

Once the relation between the body-fixed Cartesian coordinates and the three active coordinates is derived based on the above transformations, the contravariant components of 
the metric tensor $\mathbf{G}$ are obtained (see the Appendix B and Ref. $\frac{58}{50}$ for additional details); its elements will be indicated as $G^{i j}$, with $i, j$ labelling the coordinates. As usual, the resulting metric tensor is non-diagonal and depends on the active coordinates; a possible expression of (the deformation part of) the kinetic energy operator, $\hat{\mathcal{K}}_{d e f}$, for $n$ active coordinates $\mathbf{Q}=\left\{Q_{1} \ldots Q_{n}\right\}$, and for zero total angular momentum, is

$$
\hat{\mathcal{K}}_{d e f}=\sum_{i \leq j} f_{2}^{i j}(\mathbf{Q}) \frac{\partial^{2}}{\partial Q_{i} \partial Q_{j}}+\sum_{i} f_{1}^{i}(\mathbf{Q}) \frac{\partial}{\partial Q_{i}}+V_{e p}(\mathbf{Q})
$$

The symbols $f_{2}^{i j}(\mathbf{Q}), f_{1}^{i}(\mathbf{Q})$ and $V_{e p}(\mathbf{Q})$ stand for functions of active coordinates, and they are obtained from the contravariant components of the metric tensor. $V_{e p}(\mathbf{Q})$ is a scalar term often called extrapotential $\left[\frac{58}{60}\right.$ and it may appear when a non-Euclidean volume element, $d \tau_{d e f}=\rho(\mathbf{Q}) \cdot d Q_{1} \cdot d Q_{2} \cdots d Q_{n}$, is used, i.e., when $\rho(\mathbf{Q})$ is not the Jacobian of the coordinates transformation.

The results presented below can be considered complementary to previous dynamical studies based on model PESs for the isomerization in retinals $21|22| 25 \mid 26$ that assume a constant diagonal form of the metric tensor due to the fact that its components are fitted to reproduce spectroscopy data. In order to be able to directly compare our quantum dynamics results with the two-dimensional Hahn-Stock model ${ }^{[56}$ and to the quantum-classical simulations performed in the following sections, we construct as well approximate kinetic energy operators where the metric tensor is either constant or constant and diagonal. The constant metric tensor is determined by evaluating the position-dependent metric tensor over 45 geometries, spanning homogeneously the configuration space, and averaging its components; the constant and diagonal metric tensor is determined by neglecting the off-diagonal components of the constant metric tensor. It is worth noting that, due to the symmetry, averaging the elements $G^{r \theta}$ and $G^{r \phi}$ yields zero. The diagonal components of the contravariant metric tensor can be identified as the inverse of an effective mass and moments of inertia associated, respectively, to the three curvilinear coordinates, BLA, Tors, and HOOP; their values are 
reported in Table 1 .

Table 1: Constant components of the contravariant metric tensor obtained by averaging the exact position-dependent metric tensor over 45 geometries (in atomic units). Only the non-zero values are given.

\begin{tabular}{c|c} 
Component & Value in a.u. \\
\hline \hline$G^{r r} \equiv 1 / M_{r}$ & $7.981 \cdot 10^{-5}$ \\
$G^{\theta \theta} \equiv 1 / I_{\theta}$ & $2.599 \cdot 10^{-5}$ \\
$G^{\phi \phi} \equiv 1 / I_{\phi}$ & $40.375 \cdot 10^{-5}$ \\
$G^{\theta \phi}$ & $4.025 \cdot 10^{-5}$
\end{tabular}

\subsection{Probabilities and quantum yield}

In order to easily compare quantum dynamics results based on the three forms of the metric tensor we focus here on the probabilities of the cis and trans conformers in the $S_{0}$ and $S_{1}$ adiabatic states as functions of time. To this end we will analyze the time evolution of:

- the populations of the ground and first-excited states in the three-dimensional model and in the two-dimensional Hahn-Stock model for the cis and trans conformers;

- the quantum yield in the three-dimensional model and in the two-dimensional HahnStock model.

The dynamics is initiated in $S_{1}$ at the cis geometry so as to reproduce the effect of an ultra-short photo-excitation that takes the molecule from $S_{0}$ to the first spectroscopic state without geometrical modifications. Therefore, the initial vibrational wavepacket is a threedimensional Gaussian centered at the minimum of the ground-state PES (additional details are given in Appendix C). We compare our results to the Hahn-Stock model,,$[56$ which is based on the parametrization of the $S_{0}$ and $S_{1}$ PESs as functions of a reactive torsion coordinate and of a collective vibrational coordinate that couples $S_{0}$ and $S_{1}$. The protocol illustrated above for the initialization of the dynamics is employed to compute the corresponding probabilities in this two-state two-dimensional model. It is worth stressing that the 2D Hahn-Stock model is not an analytical model for PSB3, but rather for "the photoinduced 11-cis $\rightarrow$ all-trans 
isomerization of retinal in rhodopsin". 56 Thus, the used coordinates are to be considered as "effective modes" and do not correspond exactly to the Tors and BLA coordinates of the three-dimensional model.

The probabilities of finding cis and trans conformations either in the $S_{0}$ or $S_{1}$ states are defined as

$$
\begin{aligned}
P_{\text {cis }}^{S_{i}}(t) & =\iiint_{|\theta|<90^{\circ}}\left|\chi^{S_{i}}(\theta, r, \phi, t)\right|^{2} d r d \phi d \theta \\
P_{\text {trans }}^{S_{i}}(t) & =\iiint_{|\theta|>90^{\circ}}\left|\chi^{S_{i}}(\theta, r, \phi, t)\right|^{2} d r d \phi d \theta,
\end{aligned}
$$

where the nuclear probability density in state $S_{i}($ with $i=0,1)$ is indicated as $\left|\chi^{S_{i}}(\theta, r, \phi, t)\right|^{2}$. A similar expression is used for the two-dimensional model, clearly without the dependence on the HOOP coordinate ( $\phi$ in the equations). The population of state $S_{i}$ is given by

$$
P^{S_{i}}(t)=P_{\text {cis }}^{S_{i}}(t)+P_{\text {trans }}^{S_{i}}(t) .
$$

Figure 2 shows that all quantum simulations based on the three-dimensional model predict that in 200 fs the $S_{1}$ population tends to about 0.5 , which is arguably due to the reduced dimensionality of the system from many (36 in PSB3 and 156 in rPSB11) to 3 modes. Also, an ultrafast decay of the $S_{1}$ probability towards the photo-products already at $50 \mathrm{fs}$ is common to all results based on the three-dimensional model. We notice quantitative differences between exact results (black lines) and the two approximations of the metric tensor, i.e., constant (dark-green lines) and diagonal-constant (light-green lines). However, qualitatively, the behavior of the probabilities as functions of time is not affected by the approximations, in particular by the use of a diagonal and constant metric tensor. This results justifies its use in the quantum-classical simulations presented in Sections 3 and 4. We notice oscillations in the probabilities of $S_{1}$-trans (upper right panel of Fig. 2), $S_{0}$-cis (lower left panel of Fig. 2) and $S_{0^{-}}$trans (lower right panel of Fig. 22) that appear as soon as 
cis conformer

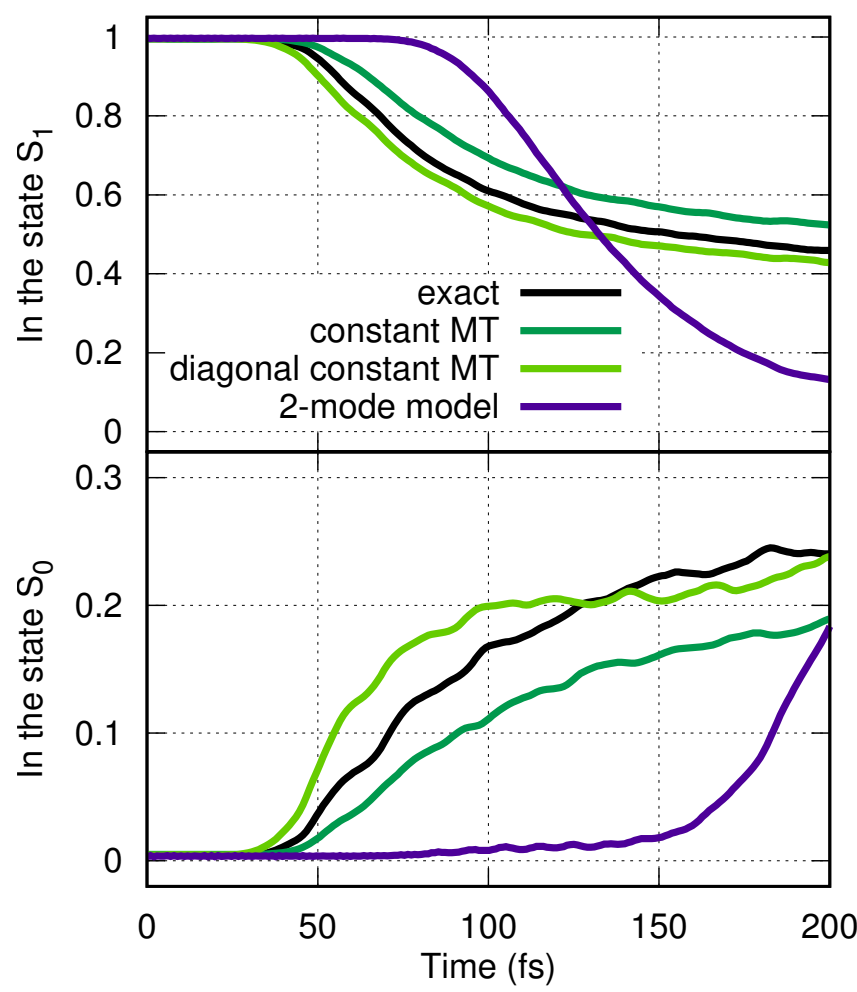

trans conformer

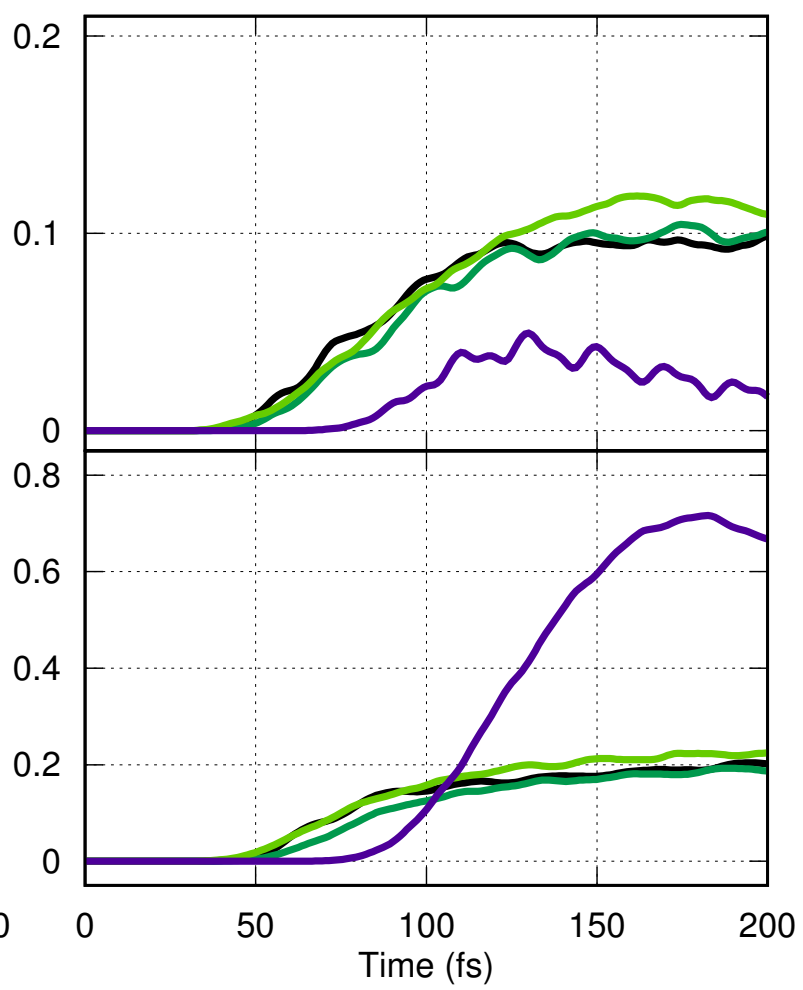

Figure 2: Probabilities of the cis (left panels) and trans (right panels) conformers in the ground state $S_{0}$ (lower panels) and in the excited state $S_{1}$ (upper panels) as functions of time. Exact quantum results for the three-dimensional model, using a non-diagonal positiondependent metric tensor (MT), are shown in black. Quantum results using a constant MT are shown in dark green, and quantum results using a diagonal constant MT are shown in light green. The corresponding probabilities determined based on the Hahn-Stock twodimensional model ${ }^{[56}$ are shown in purple.

the curves (black, green and light-green) increase from zero at around $50 \mathrm{fs}$. This feature will be discussed later on, since an analogous behaviour will be observed as well in the plot of the quantum yield (in Fig. 3) and in the plot of the kinetic energy along the HOOP coordinate (in Fig. 4) as functions of time.

The results of the two-dimensional model (purple lines) are qualitatively different from the results of the three-dimensional model. The decay of the $S_{1}$-cis probability (mainly) in favour of the $S_{0}$-trans is delayed if compared to the results of the three-dimensional potentials (in the three-dimensional model, $S_{1}$-to- $S_{0}$ relaxation through the CI is clearly ultrafast). More specifically, the two-dimensional model ignores the effect of the HOOP 
mode, and in particular of the correlation between HOOP and Tors (see analysis presented in Section 4) in preventing/favouring the photo-product formation.

Based on the probabilities just analyzed, we can estimate the quantum efficiency, or quantum yield, of the process. Here, we define the quantum yield as the ratio between the trans products and all products of the reaction, that is

$$
Q Y(t)=\frac{P_{\text {trans }}^{S_{0}}(t)+P_{\text {trans }}^{S_{1}}(t)}{P_{\text {cis }}^{S_{0}}(t)+P_{\text {trans }}^{S_{0}}(t)+P_{\text {trans }}^{S_{1}}(t)} .
$$

The quantum efficiency as function of time is shown in Fig. 3, where quantum results based

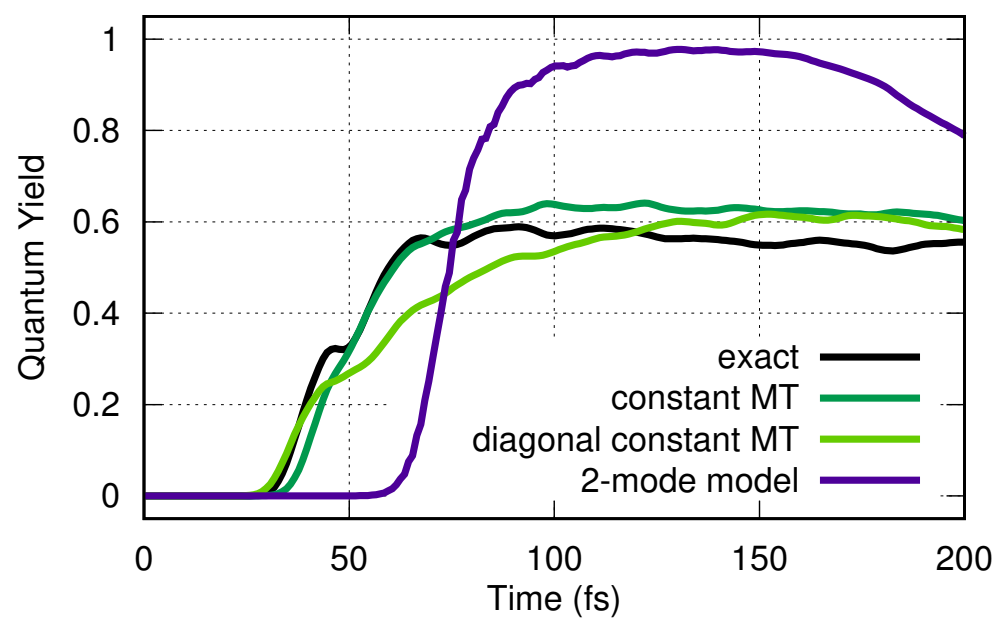

Figure 3: Quantum yield as function of time estimated from Eq. (8). The color code is the same as in Fig. 2,

on our three-dimensional model ${ }^{[27}$ are compared to the Hahn-Stock model. $[56$ Once again we observe a qualitative difference between the two models, whereas a qualitative agreement clearly persists between exact and approximate (quantum) predictions for the threedimensional model PESs, from which we can extract a long-time estimate of the quantum efficiency of about 0.6 .

\subsection{Nuclear kinetic energy}

It is instructive to further analyze quantum dynamics based on the three-dimensional model ${ }^{27}$ by looking at the average nuclear kinetic energy along BLA, Tors and HOOP as function of 
time evaluated numerically with TNUM. $\frac{58}{1}$ This analysis

- confirms that using a diagonal-constant metric tensor is a good approximation for the exact dynamics;

- relates the oscillations observed in Section 2.1 in the probabilities of $S_{1}$-trans, $S_{0^{-}}$cis, and $S_{0}$-trans to the oscillations along HOOP;

- highlights similarities and differences between the BLA mode of the three-dimensional model and the effective stretching mode of the two-dimensional model;

- points out a qualitative different behavior of the reactive modes in the two models.

The first observation is crucial to validate the following comparison of the nuclear kinetic energy between quantum and quantum-classical results. In fact, the general expression of the nuclear kinetic energy operator contains "off-diagonal" contributions describing the coupling between the modes. Those couplings are completely neglected when the diagonal-constant form of the metric tensor is used, as is the case in quantum-classical simulations presented later on.
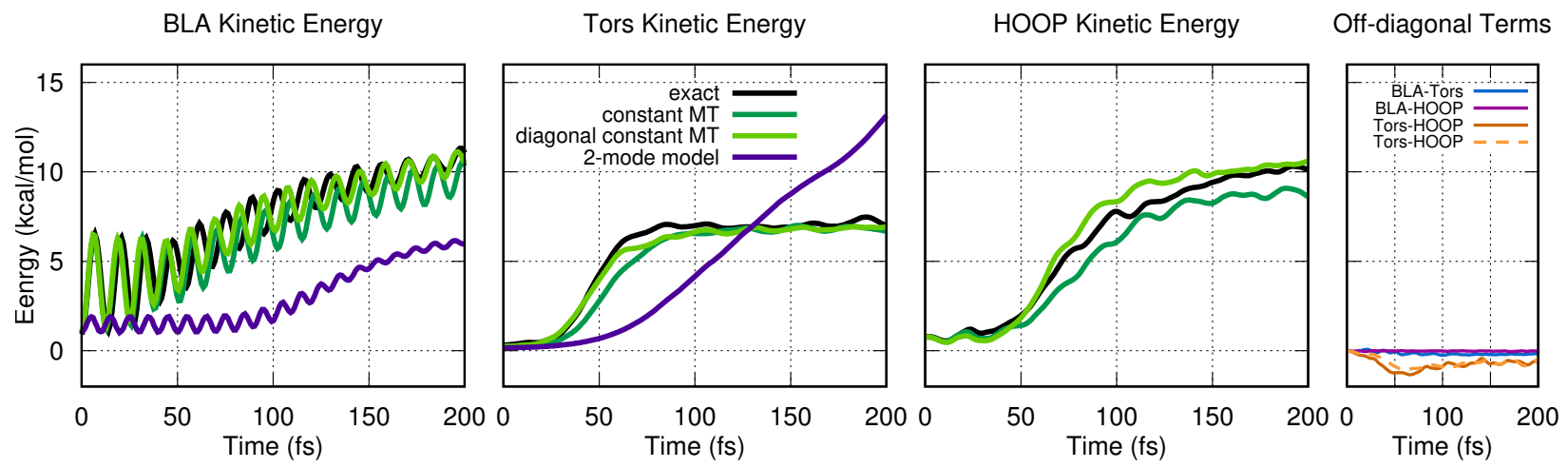

Figure 4: Nuclear kinetic energy (diagonal) contributions along BLA, Tors and HOOP as functions of time. The color code is the same as in Fig. 2. The right panel shows the offdiagonal contributions $\left(f_{2}^{i j}(\mathbf{Q}) \frac{\partial^{2}}{\partial Q_{i} \partial Q_{j}}\right.$ for $i \neq j$ in Eq. 40$)$ arising from the coupling of the modes: continuous lines refer to exact calculations; the dashed line refers to the calculations based on the constant metric tensor.

Figure 4 shows the expectation value of the kinetic energy operator $\hat{\mathcal{K}}_{\text {def }}$ on the instantaneous vibronic state decomposed into its "diagonal" BLA, Tors and HOOP contributions 
for exact (black lines) calculations, and for the two approximations based on the constant metric tensor (dark-green lines) and diagonal-constant metric tensor (light-green lines). The overall agreement between exact and approximate results is satisfactory to justify the use of a diagonal-constant metric tensor in the following calculations based on trajectories, even though a small dephasing is observed between 50 and approximately 120 fs along the BLA coordinate. When the metric tensor is non-diagonal, kinetic energy contributions arising from the coupling between the modes are observed (off-diagonal terms in Fig. 4). In particular, all modes are coupled in the exact treatment, even though not strongly, as the continuous lines in the right panel of Fig. 4 show, whereas only Tors and HOOP are weakly coupled when the constant metric tensor is used, as shown by the dashed line in the right panel of Fig. 4 (the coupling with BLA is identically zero in this case, since the components $G^{r \theta}$ and $G^{r \phi}$ of the constant metric tensor are zero). Nonetheless, these additional (off-diagonal) contributions are negligible if compared to the diagonal terms. Note, also, that each term of the nuclear kinetic energy operator of Eq. (4) is in general not Hermitian and, therefore, its expectation value can be complex. It is only the sum of all terms that is real. However, all imaginary parts are close to zero in all cases and, thus, they are not shown here.

As pointed out in Section 2.1, characteristic oscillations are observed in Figs. 2 and 3 when the curves corresponding to the three-dimensional model increase from zero at around 50 fs. A similar behavior is observed in the curves representing the kinetic energy along HOOP as function of time. Therefore, it is plausible to conclude that those oscillations in the probabilities (Fig. 2) are induced by the HOOP motion. Furthermore, the oscillations persist in the three forms of the metric tensor, even when the kinetic coupling between modes is switched off, indicating that they are solely an effect of the PESs.

As for the results shown in Section 2.1, dynamics performed with the Hahn-Sock twodimensional model shows qualitative differences with respect to our model when analyzing the kinetic energy as function of time. In Fig. 4 we directly compare the kinetic energy along BLA with the kinetic energy along the coupling mode of the two-dimensional model 
(left panel). This choice is justified based on the definition of the coupling mode given in Ref.:56 "a delocalized stretching motion of the polyene chain, whereby single and double bonds interchange". The kinetic energy along this coupling mode oscillates with a period of about $10 \mathrm{fs}$ and increases after $100 \mathrm{fs}$, with amplitudes that are damped at long times. Such a behavior suggests that vibrational coherence is lost while the nuclear wavepacket is transferred from $S_{1}$ to $S_{0}$ acquiring kinetic energy along the reactive torsional coordinate (Tors Kinetic Energy panel in Fig. 4). However, the increase of kinetic energy along the coupling mode is delayed in comparison to the increase along BLA, appearing just after $50 \mathrm{fs}, 6$ while the period of the oscillations is similar (about $13 \mathrm{fs}$ for the three-mode dynamics). The comparison of the Tors kinetic energy for the two models shows another qualitative difference: in the two-dimensional model the curve does not reach a plateau within the simulated 200 fs.

In Section 2.1 and in the present section, we have shown that the two-dimensional model PESs yield, in general, different dynamics from the three-dimensional model. Therefore, in the following sections, we will not pursue the comparison between the two models based on quantum-classical simulations.

To conclude this section, let us define the expression of the nuclear kinetic energy operator when the diagonal-constant metric tensor is used, namely

$$
\hat{\mathcal{K}}_{\text {def }}=\frac{\hat{P}_{r}^{2}}{2 M_{r}}+\frac{\hat{P}_{\theta}^{2}}{2 I_{\theta}}+\frac{\hat{P}_{\phi}^{2}}{2 I_{\phi}}
$$

which will be used below when comparing quantum and quantum-classical results. As defined in Table 1, $M_{r}, I_{\theta}, I_{\phi}$ are the mass and moments of inertia associated to BLA, Tors and HOOP, respectively. 


\section{Analysis of the model based on quantum-classical sim-}

\section{ulations}

In Section 2, our three-dimensional model has been analyzed based on the propagation of vibronic wavepackets. We have compared those results with the two-dimensional HahnStock model, $\stackrel{56}{5}$ pointing out some qualitative/quantitative differences, in the probabilities and quantum efficiency. Furthermore, we have validated the use of a diagonal-constant metric tensor in the quantum simulations, justifying its suitability in quantum-classical simulations as well.

In this section, we will

- comment on the quantum, i.e., vibronic wavepacket, dynamics, based on the analysis of the topology of the adiabatic PESs;

- evaluate the ability of CT-MQC trajectories to reproduce the quantum evolution.

Later on, in Section 3.1, we will compute time-dependent probabilities, quantum yields, and nuclear kinetic energy, based on the trajectory-based schemes Ehrenfest, TSH, TSHEDC and CT-MQC. This extensive comparison of quantum and quantum-classical results, together with the analysis of the trajectories presented in Section 4, will provide further insights into the capability of the three-dimensional PESs to capture critical features of the microscopic mechanism of the cis-trans isomerization in PSB3 (and potentially in rPSB11), since it allows us to make connections with results from the literature based on atomistic simulations. In addition, we will be able to shed light on the properties of the used quantumclassical, approximate schemes, since the same "force field" is employed for all calculations.

Quantum (with diagonal-constant metric tensor) and quantum-classical dynamics are initiated in $S_{1}$, and classical initial conditions are Wigner-sampled from the quantummechanical nuclear probability distribution (see the Appendix $\mathrm{C}$ for details). After the initial photo-excitation, the resulting reduced nuclear densities and CT-MQC trajectories 
are shown in Fig. 5 as functions of the Tors-BLA (left panels) and Tors-HOOP (right panels) coordinates, projected on the two electronic states. The Tors-BLA reduced nuclear probability density in the $S_{i}(i=0,1)$ electronic state $\left|\chi_{\text {red }}^{S_{i}}(\theta, r, t)\right|^{2}$ is determined from the full probability density $\left|\chi^{S_{i}}(\theta, r, \phi, t)\right|^{2}$ in that state as

$$
\left|\chi_{\text {red }}^{S_{i}}(\theta, r, t)\right|^{2}=\int\left|\chi^{S_{i}}(\theta, r, \phi, t)\right|^{2} d \phi
$$

and a similar expression is used for the Tors-HOOP reduced nuclear probability density $\left|\chi_{\text {red }}^{S_{i}}(\theta, \phi, t)\right|^{2}$. We recall here that the full density is simply the sum of the contributions in $S_{0}$ and in $S_{1}$. In CT-MQC calculations, in order to establish if a trajectory is associated to $S_{0}$ or $S_{1}$, we look at the values of the electronic coefficients along that trajectory. In CT-MQC, the time-dependent electronic state along a nuclear trajectory $\mathbf{R}_{\alpha}$ is represented in the adiabatic basis $\left|S_{i} ; \mathbf{R}_{\alpha}\right\rangle$,

$$
\left|\Phi_{\mathbf{R}_{\alpha}}(t)\right\rangle=C_{\alpha}^{S_{0}}(t)\left|S_{0} ; \mathbf{R}_{\alpha}\right\rangle+C_{\alpha}^{S_{1}}(t)\left|S_{1} ; \mathbf{R}_{\alpha}\right\rangle
$$

The above-mentioned electronic coefficients are $C_{\alpha}^{S_{0}}(t)$ and $C_{\alpha}^{S_{1}}(t)$, whose squared moduli give information about the electronic populations along the trajectory $\alpha$. We have used the symbol $\mathbf{R}_{\alpha}$ for $\left(\theta_{\alpha}, r_{\alpha}, \phi_{\alpha}\right)$, i.e., the values of the nuclear coordinates for the trajectory $\alpha$. To associate a trajectory to an electronic state in Fig. 5, we made the following choice: if $\left|C_{\alpha}^{S_{i}}(t)\right|^{2} \geq 0.6$, the trajectory $\alpha$ at time $t$ is associated to the state $S_{i}$; if $0.4<\left|C_{\alpha}^{S_{i}}(t)\right|^{2}<0.6$, the trajectory $\alpha$ at time $t$ is associated to both states.

The adiabatic PESs, shown in Fig. 5 as contour plots, have mirror-plane symmetry with respect to the clockwise and couterclockwise Tors directions when the energies are plotted along Tors-BLA with HOOP $=0$ (they are $2 \pi$-periodic and symmetric with respect to Tors $=0)$. It has instead an inversion-point symmetry $\left(\mathrm{HOOP}=0\right.$, Tors $= \pm n \cdot 180^{\circ}$ with $n$ integer) when the energy is plotted along the Tors-HOOP for any BLA value. Also notice that the equilibrium conformations of PSB3 at the reactant and product minima of the $S_{0}$ 

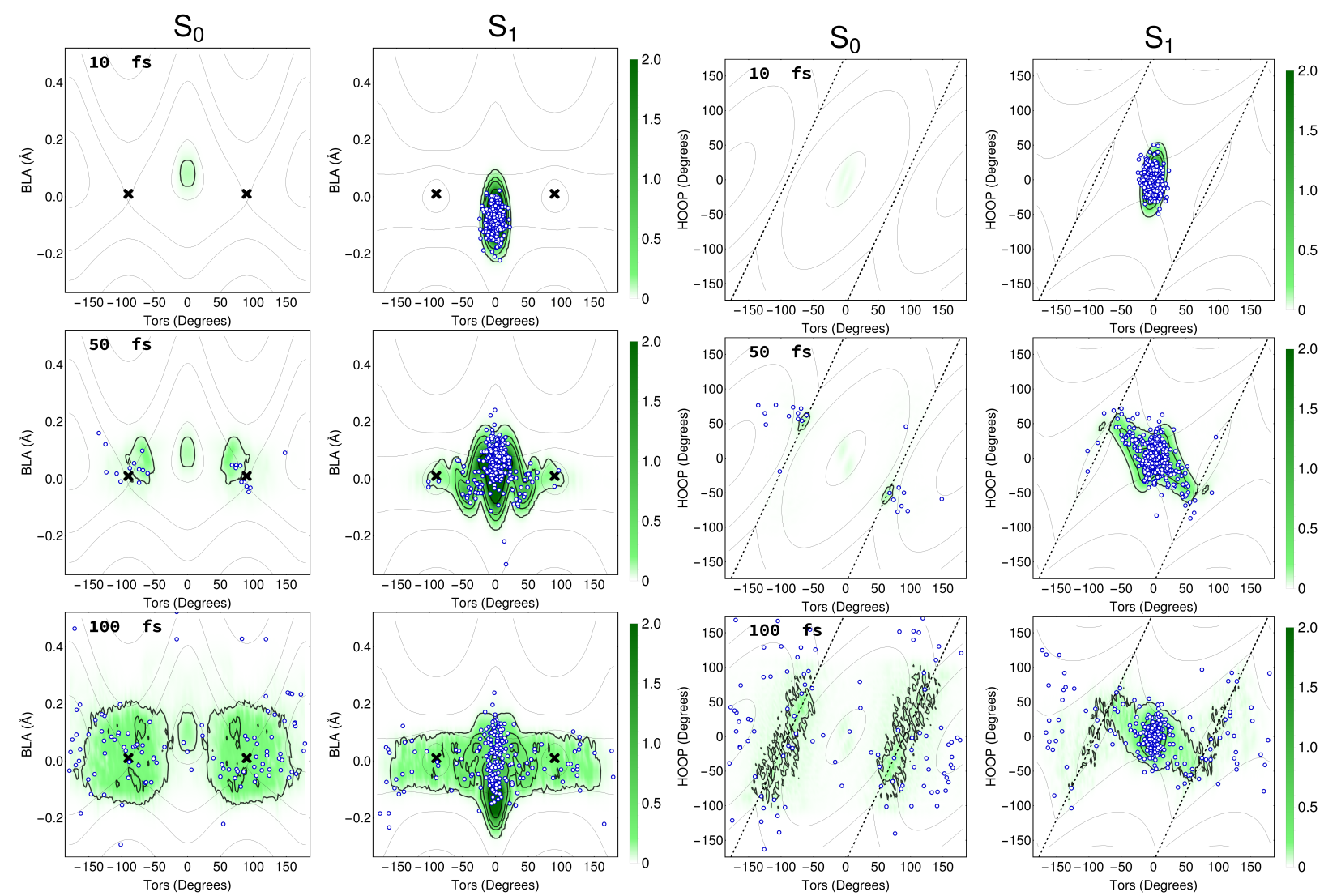

Figure 5: Adiabatic reduced nuclear densities (green areas) and distribution of CT-MQC trajectories (blue-white dots). Adiabatic PESs are represented via grey iso-contours, for $\mathrm{HOOP}=0$ on the left and for BLA $=0$ on the right. Left panels: nuclear densities in the reduced Tors-BLA space in the $S_{0}$ (left column) and $S_{1}$ (right column) states, after 10 fs (top), $50 \mathrm{fs}$ (middle), and $100 \mathrm{fs}$ (bottom) from the photo-excitation. The crosses indicate the positions of the minimum-energy CIs. Right panels: same as the left panels, with densities and trajectories represented in the reduced Tors-HOOP space. The intersection space is shown as dashed lines, for which the condition Tors $-\mathrm{HOOP} / 2 \pm 90=0$ holds.

PES are planar $\left(\mathrm{HOOP}=0\right.$, Tors $=0$ or Tors $\left.= \pm 180^{\circ}\right)$. Those features are responsible for the different and symmetric behavior during the relaxation process observed for the PSB3 model here when compared to the dynamics of rPSB11 which occurs in a completely asymmetric (actually chiral) Rh environment, pushing the entire molecular population along an exclusively counterclockwise torsional motion (see for instance Ref. ${ }^{7}$ ).

In Fig. 5, the vibrational wavepacket is shown at three time steps during the evolution, with CT-MQC trajectories following closely the (reduced) densities. On the left panels, the Tors-BLA reduced density on $S_{1}$ shows long-lasting vibrations along the BLA coordinate 
accompanied, already before $50 \mathrm{fs}$, by fast funneling towards the trans product $\frac{\text { t38 }}{\text { through }}$ the CIs located in the region Tors $= \pm 90^{\circ}$, BLA $=0$. The model does not take into consideration either additional (internal) vibrational modes or the environment, and due to the reactant planarity, population transfer from $S_{1}$ to $S_{0}$ takes place symmetrically along the clockwise and counterclockwise directions Tors $>0$ and Tors $<0$. Furthermore, the energy pumped into the system via the initial electronic excitation cannot be dissipated, causing the nuclear wavepacket to remain localized in the CI region. The effect is that $S_{1}$ population decay is slower than that expected in the fully-atomistic PSB3 system with 36 vibrational degrees of freedom, and continuous transfer from $S_{1}$ to $S_{0}$ and $S_{0}$ to $S_{1}$ eventually stabilizes the $S_{1}$ and $S_{0}$ populations at long times around 0.5. $2^{2}$

The observed motion along the Tors and HOOP modes is correlated. ${ }^{[37}$ Once the torsional mode is activated, the wagging helps the $S_{1}$ population to reach the intersection space by breaking the bonding character of the $\pi$-system along the $\mathrm{C}_{2}=\mathrm{C}_{3}$ reactive double bond. In fact, the most favorable path towards the degeneracy points is the path that sets to zero the $\pi$-system overlap, which is approximately proportional to the geometrical variable Tau $=$ Tors $-\mathrm{HOOP} / 2$ (see Fig. 1, right panel). The overlap is thus assumed to be zero at Tau $= \pm 90^{\circ}$, and reaches a maximum at Tau $=0$ and Tau $= \pm 180^{\circ}$. The Tors-HOOP reduced probability density transfers from $S_{1}$ to $S_{0}$ following the encounter with the intersection space located at values close to Tau $= \pm 90^{\circ}$. We noticed that the density/trajectories moving towards Tors $= \pm 180^{\circ}$ (as shown in Fig. 5, the $S_{0}$ PES reactant and product minima have $\mathrm{HOOP}=0$ ) are able to reach the trans-PSB3 product only when certain relationships between the phase of the Tors and HOOP velocities are satisfied when passing through the decaying region. This point will be discussed later on the basis of a trajectory analysis.

\footnotetext{
${ }^{2}$ Note that an analogous stabilization of the populations around 0.5 is observed as well for the twodimensional model discussed in Section 2 towards $500 \mathrm{fs}$.
} 


\subsection{Probabilities and quantum yield}

Detailed information about the $S_{1}$-to- $S_{0}$ relaxation mechanism accompanying the cis-trans isomerization can be extracted from the analysis of the time-dependent probabilities, as done for quantum-dynamics simulations in Fig. 2. We report here the corresponding results obtained from the quantum-classical propagations and we point out some differences

- in early-time dynamics between quantum and quantum-classical results;

- among the quantum-classical schemes themselves.

In Ehrenfest and CT-MQC calculations, the cis and trans configuration, i.e., the reactant and product structure, probabilities of Eqs. (5) and (6) are estimated as

$$
\begin{aligned}
P_{\text {cis }}^{S_{i}}(t) & \simeq \frac{1}{N_{\text {traj }}} \sum_{\alpha=1}^{N_{\text {traj }}}\left|C_{\alpha}^{S_{i}}(t)\right|^{2}\left(1-H\left(\left|\theta_{\alpha}\right|-90^{\circ}\right)\right) \\
P_{\text {trans }}^{S_{i}}(t) & \simeq \frac{1}{N_{\text {traj }}} \sum_{\alpha=1}^{N_{\text {traj }}}\left|C_{\alpha}^{S_{i}}(t)\right|^{2} H\left(\left|\theta_{\alpha}\right|-90^{\circ}\right),
\end{aligned}
$$

respectively, while in TSH and TSH-EDC we simply count the number of trajectories in $S_{i}$ either with values $\mid$ Tors $\mid<90^{\circ}$ (cis) or with values $\mid$ Tors $\mid>90^{\circ}$ (trans). In Eqs. (12) and (13) the symbol $H$ stands for the Heaviside step function, that is equal to 1 if its argument is positive and equal to 0 if its argument is negative $(H(x)=1$ if $x>0$ and $H(x)=0$ if $x<0)$.

Figure 6 shows the comparison between quantum results, employing the diagonal-constant metric tensor (light-green lines), and quantum-classical results obtained based on Ehrenfest dynamics (purple lines), CT-MQC (blue lines), TSH (red lines), and TSH-EDC (orange lines). While the decay of the probability of the cis conformer in $S_{1}$ (upper left panel of Fig. 6) is well captured by all quantum-classical schemes, some disagreement is observed between quantum and TSH/TSH-EDC results in the behavior of the probability of producing the trans conformer in the excited state already after 50 fs (upper right panel of Fig. 6), as well as between quantum and all quantum-classical schemes in the probability of producing 
cis conformer

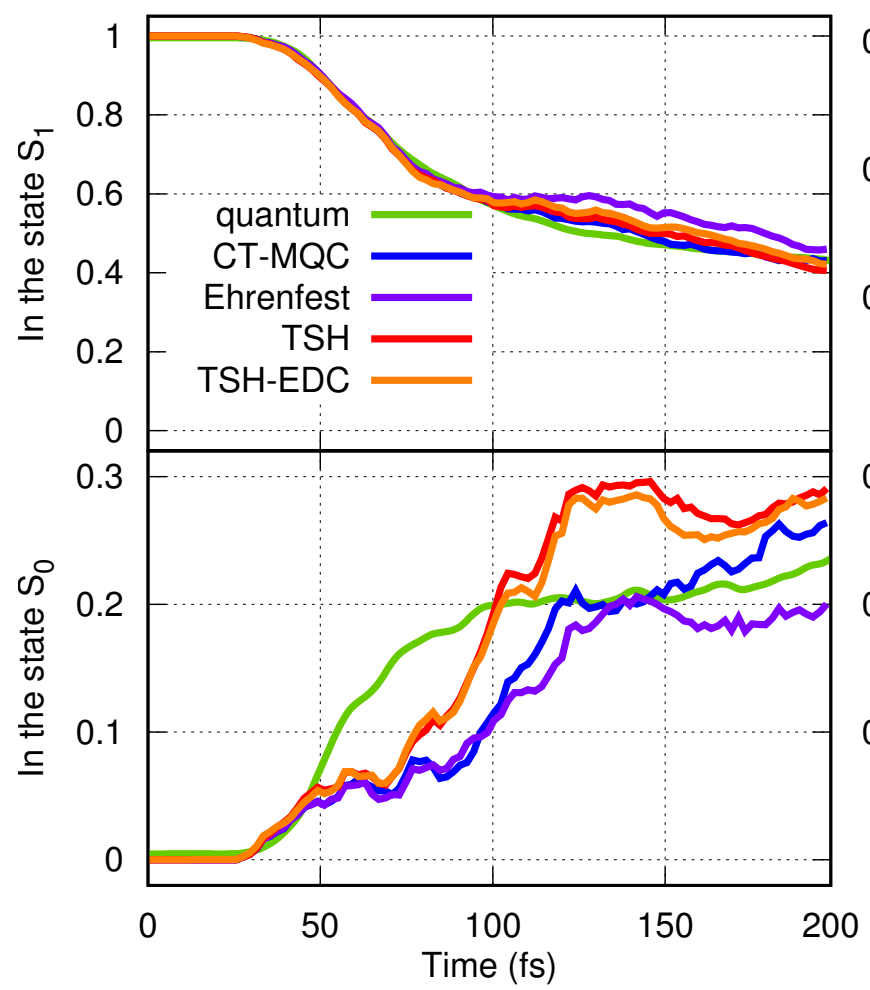

trans conformer

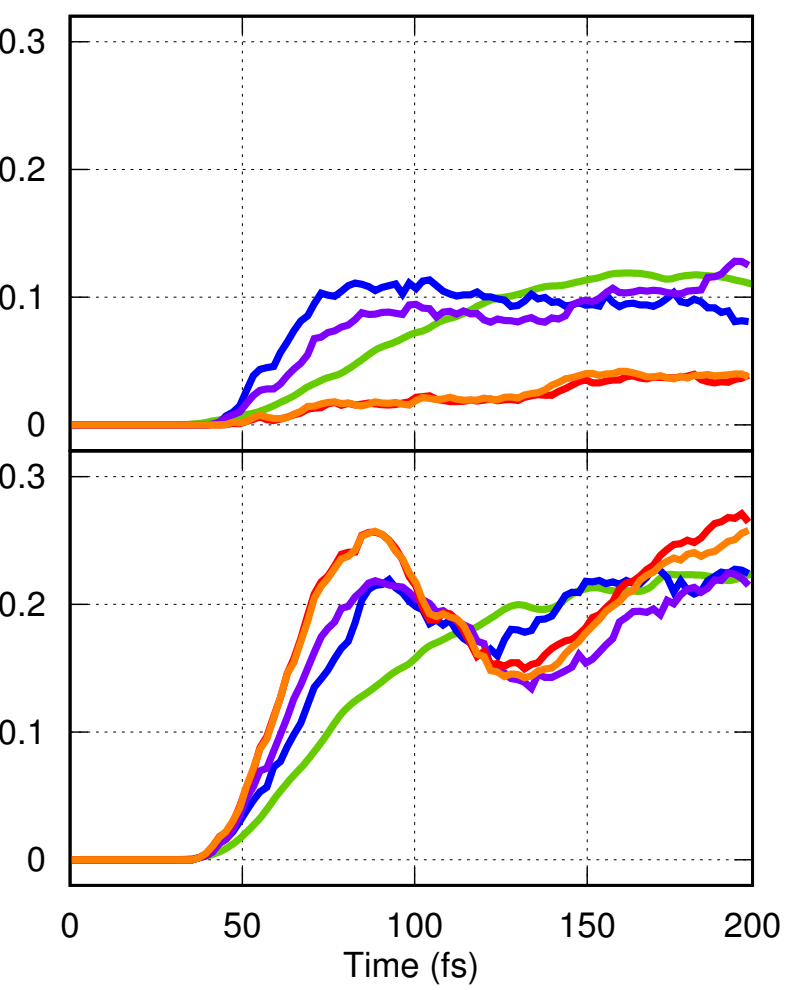

Figure 6: Probabilities of the cis (left panels) and trans (right panels) conformers in the ground state $S_{0}$ (lower panels) and in the excited state $S_{1}$ (upper panels) as functions of time. Quantum-classical results based on Ehrenfest dynamics (purple lines), CT-MQC (blue line), TSH (red lines) and TSH-EDC (orange lines) are compared to quantum results using the diagonal-constant metric tensor (light-green lines).

the trans conformer in the ground state at around $80 \mathrm{fs}$ (lower right panel of Fig. 6). The larger population of the trans conformer produced in $S_{0}$ predicted by the trajectory-based schemes is somehow compensated at around $80 \mathrm{fs}$ by an inversion of that behavior in the cis conformer in $S_{0}$. At long times, Ehrenfest and CT-MQC seem to capture the behavior of the probabilities in better agreement with quantum results than TSH/TSH-EDC: for Ehrenfest and CT-MQC, the trans- $S_{1}$ and cis- $S_{0}$ probabilities stabilize around the quantum result, whereas the oscillations in the trans- $S_{0}$ probabilities have slightly smaller amplitude than in TSH(-EDC). Note also that, despite the inclusion of decoherence corrections in TSH-EDC, the oscillations of the probability of the trans conformer in $S_{0}$ persist. Such oscillations are significantly reduced in CT-MQC. 


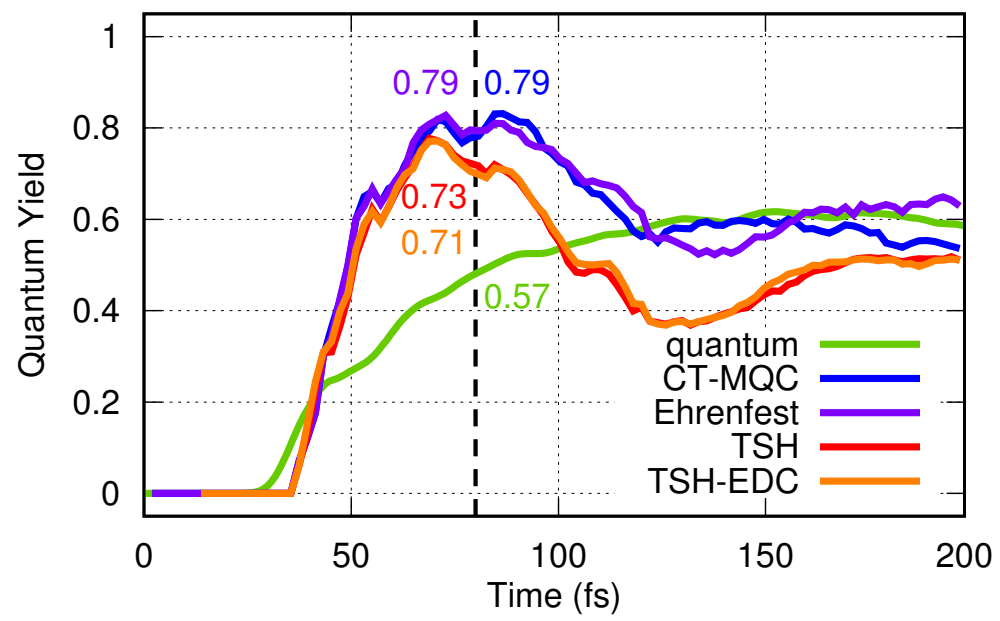

Figure 7: Quantum yield as function of time estimated from Eq. (8). The color code is the same as in Fig. 6. Numerical values at $80 \mathrm{fs}$ are explicitly indicated (dashed vertical line).

The overestimation - with respect to the quantum results - of the probability of forming the trans-PSB3 product in $S_{0}$ at around $80 \mathrm{fs}$, i.e., immediately after reaching the intersection space region at about $50 \mathrm{fs}$ delay, is common to all quantum-classical methods. ${ }^{3}$ It appears that trajectories are funneled through the intersection space at a higher rate than the quantum wavepacket, resulting in a significantly overestimated quantum efficiency at short time scales. To show this, we present in Fig. 7 the quantum efficiency, or quantum yield, from Eq. (8). The computed quantum-classical quantum yield is overestimated at short time, by about $25-35 \%$ depending on the method (see the values at 80 fs reported in Fig. 7), if compared to quantum predictions. Since QM/MM simulations are usually performed based on TSH-EDC, this result might suggest a similar overestimation of the quantum efficiency in fully-atomistic studies. However, we also expect that taking into account larger number of vibrational degrees of freedom might severely suppress the trans product thermal backisomerization as well as the return to the $S_{1}$ state, which, according to the data in Fig. 5 , contribute to decrease the formation of the product. It is interesting to notice that, while

\footnotetext{
${ }^{3}$ Similar disagreements have been ascribed in the literature to the incorrect account of geometric-phase effects. $\frac{61}{}$ Even though quantum calculations are performed in the diabatic basis and quantum-classical simulations in the adiabatic basis, we believe that geometric-phase effects are not at the origin of the disagreement. In fact, even if geometric-phase effects are not accounted for in trajectory-based schemes, the local nature of the trajectories somehow helps in circumventing issues that can be related to them, as explicitly proven for Ehrenfest and TSH dynamics in recent studies. ${ }^{62 \mid 63}$
} 
the quantum yield predicted by TSH and TSH-EDC is lower than the expected - quantum - value at around 120 fs, Ehrenfest and CT-MQC predict a rather good behavior towards the value of 0.6 at the same time.

We notice that TSH results reported in Figs. 6 and 7 are very similar to TSH-EDC results, while they are different form Ehrenfest and CT-MQC. The decoherence-correction strategy used in TSH-EDC ${ }^{44}$ damps exponentially the electronic population that is not associated to the active state along each trajectory over a certain characteristic time. However, due to the nature of the model PESs, at long times the trajectories remain localized in the vicinity of the intersection space, therefore, the decoherence correction is not able to "stabilize" to zero the electronic population associated to the non-active state far from the intersection region. Here, by "nature of the model PESs", we mean the extent of the degeneracy regions of the $S_{0}$ and $S_{1}$ PESs along Tors and HOOP coordinates. We expect, however, that this kind of decoherence correction performs better as the dimensionality of the problem increases. For similar reasons, it is not surprising that Ehrenfest results are close to CT-MQC results. CT-MQC equations are basically Ehrenfest equations plus a decoherence term that tracks the delocalization of the wavepackets associated to different electronic state. $\frac{45}{\text { Since the } S_{0}}$ and $S_{1}$ wavepackets delocalize over all configuration space, these decoherence effects are not strong in this case.

The "common" disagreement in early-time dynamics of all quantum-classical methods when compared to quantum results will be commented on in Section 3.2. Here, we will discuss the disagreement between Ehrenfest/CT-MQC dynamics and TSH(-EDC) dynamics. We believe that the nature of the model PESs itself is responsible for the differences in: the trans- $S_{1}$ population after $50 \mathrm{fs}$; the $c i s-S_{0}$ population after $80 \mathrm{fs}$; the trans- $S_{0}$ population mainly between 50 and 100 fs; the quantum yield between 80 and 120 fs. It has been shown in an early work on TSH,, 64 that Ehrenfest - mean-field - dynamics performs slightly better than TSH when a system presents delocalized non-adiabatic couplings; in the three-dimensional model PESs, the trajectories are found at long times in the coupling region, thus essentially 
experiencing the effect of the non-adiabatic couplings as if they were delocalized in space. Furthermore, as stated above, (i) CT-MQC equations resemble Ehrenfest equations (the difference is a term that couples CT-MQC trajectories inducing decoherence), while (ii) TSH and TSH-EDC results are similar since the used decoherence corrections are not remarkably effective in this case. These observations explain the better agreement of Ehrenfest/CT-MQC results with quantum results than $\mathrm{TSH}(-\mathrm{EDC})$.

Detailed information about the quantum-classical $S_{1}$ reaction pathways can be extracted by analyzing the trajectories. Based on such an analysis, we have observed four possible trends: (i) some trajectories remain in the cis- $S_{1}$ state; (ii) some trajectories decay from $S_{1}$ to $S_{0}$ towards the photo-product, but they reach the minimum of the $S_{0}$ PESs with large kinetic energy, such that the rotation around the reactive $\mathrm{C}_{2}=\mathrm{C}_{3}$ bond continues, eventually bringing the system through a CI region and back to $S_{1}$ either in the cis or in the trans geometry; (iii) some trajectories reach a CI region, but the combination of the Tors and HOOP velocities (see discussion in Section 4) prevents the formation of the photoproduct and delay the $S_{0}$ relaxation, such that they go back to $S_{1}$ in the cis geometry; (iv) some trajectories reach a CI region and decay to $S_{0}$, however, the combination of the Tors and HOOP velocities yields the formation of the cis conformer rather than the trans conformer. Paths (ii) and (iv) are discussed in more detail in Section 4, where the trajectoires are explicitly shown. The combination of paths (i)-(iii) yields the $S_{1}$ population of the cis conformer with the largest probability for all methods, as observed in Fig. 6, with cis-PSB3 mainly populating the $S_{1}$ state. If we instead exclusively look at the $S_{0}$ population, one can see a more similar development of the cis-PSB3 and trans-PSB3 conformations.

\subsection{Nuclear kinetic energy}

In this section we compare the nuclear kinetic energy from quantum and quantum-classical calculations in order to

- investigate the relation between the larger probability of the trans-PSB3 conformer 
formed in $S_{0}$ at short time as predicted by the quantum-classical schemes with respect to the benchmark quantum results;

- discuss the excitation energy distribution among the vibrational modes considered in the three-dimensional model.

The classical nuclear kinetic energy is averaged over the trajectories and it is decomposed along the three nuclear modes according to the expression

$$
\mathcal{K}^{(c l)}(t)=\frac{1}{N_{\text {traj }}} \sum_{\alpha=1}^{N_{\text {traj }}} \mathcal{K}_{\alpha}^{(c l)}(t)=\frac{1}{N_{\text {traj }}} \sum_{\alpha=1}^{N_{\text {traj }}}\left(\frac{P_{r, \alpha}^{2}}{2 M_{r}}+\frac{P_{\theta, \alpha}^{2}}{2 I_{\theta}}+\frac{P_{\phi, \alpha}^{2}}{2 I_{\phi}}\right)
$$

where $P_{r, \alpha}, P_{\theta, \alpha}, P_{\phi, \alpha}$ are the momenta of the trajectory $\alpha$ along BLA, Tors and HOOP.

BLA Kinetic Energy

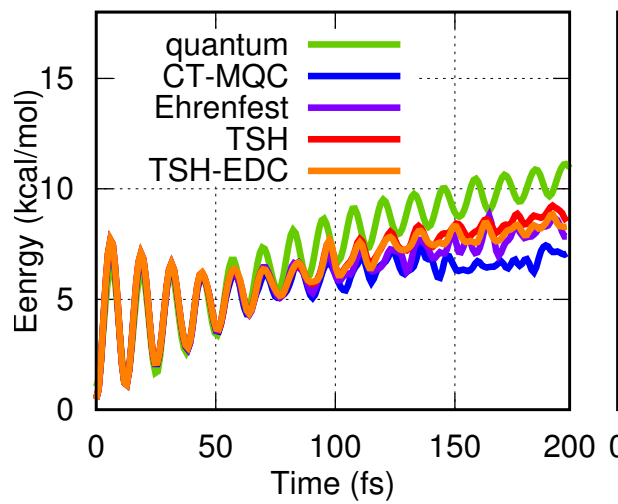

Tors Kinetic Energy

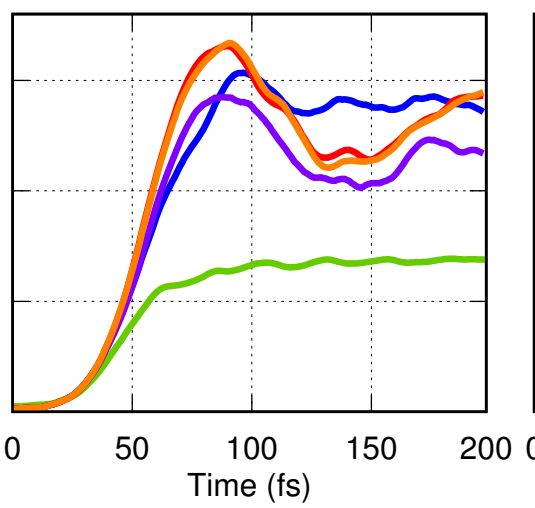

HOOP Kinetic Energy

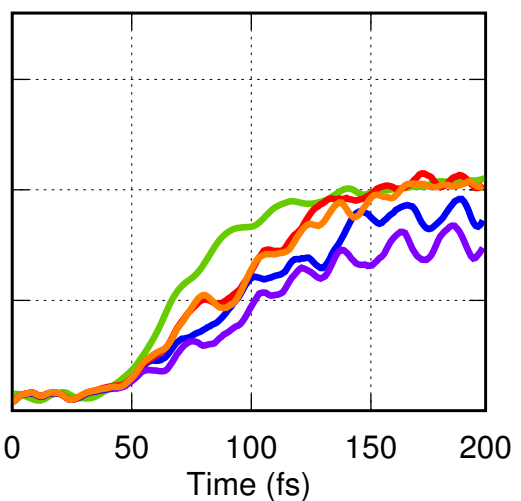

Figure 8: Nuclear kinetic energy along the BLA (left), Tors (center), and HOOP (right) coordinates, as function of time. The color code is the same as in Fig. 6 ,

Kinetic energy values from quantum and quantum-classical calculations are shown in Fig. 8. Within the first 50 fs of dynamics the amplitude of the oscillations in BLA kinetic energy described at the quantum level is damped while the wavepacket, centered around Tors $=0$, spreads towards the CIs at Tors $= \pm 90^{\circ}$. The plots indicate that the kinetic energy is mainly transferred from BLA to Tors, and in fact, in the same time interval, the Tors component increases. Afterwards, the BLA kinetic energy change can be decomposed into two contributions: a monotonic increasing contribution and an oscillating contribution with decreasing amplitude. The former can be explained by the wavepacket funneling down 
the CIs towards $S_{0}$, with following loss in potential energy. The latter is the result of the loss of vibrational coherence in the BLA direction, with the wavepacket spreading along Tors (see Fig. 5 and the Supporting Information animation of the wavepacket dynamics). It is also apparent that the funneling process is accompanied by an increase of Tors and HOOP kinetic energies, until a plateau is reached.

The largest deviation of quantum-classical results from the quantum result is observed in the Tors kinetic energy. After 50 fs the classical Tors motion acquires too much kinetic energy if compared to its quantum counterpart with a peaked difference reached at 80 fs delay. This excess energy gain is partially compensated by a parallel loss in BLA and HOOP kinetic energy, as shown in Fig. 8 (left and right panels, respectively), and partially by the potential energy decrease (not shown here) relative to the quantum result. The disagreement in the potential energy is related to the larger amount of population in the trans- $S_{0}$ state between 50 fs and 100 fs predicted by quantum-classical calculations if compared to the quantum results. A possible reason for the disagreement can be found in the nature of the quantum-classical propagation scheme itself. Initial conditions are sampled according to the quantum harmonic Wigner distribution, but classical-like equations of motion are used for the dynamics. The redistribution of the excitation energy among the modes and excess energy in Tors can be affected by this inconsistency between the preparation of the initial state and the subsequent evolution, especially in the studied conditions where the system is isolated and no channels for energy loss are considered. This issue might be reminiscent of the zero-point energy leakage observed in condensed-phase simulations accounting for nuclear quantum effects, $\stackrel{65 \mid 66}{6}$ namely the incorrect energy transfer between high-frequency and low-frequency modes.

Analyzing Fig. 8 further, we notice oscillations of BLA energy persisting at long times when described at the quantum level, but are captured by the classical methods only up to 120 fs. Tors kinetic energy predicted by CT-MQC seems to reach a plateau at long times, in agreement with quantum results; Ehrenfest, TSH and TSH-EDC, instead, present 
low-frequency oscillations up to the end of the simulated dynamics. Motion along the Tors coordinate appears to be less coherent than along BLA and HOOP, a feature that is captured by CT-MQC slightly better than the other trajectory-based methods. In fact, in CT-MQC the trajectories are coupled, which is the main difference with respect to the other quantum-classical schemes, but, as pointed out above, one sees significant Tors low frequency oscillations. Nevertheless, the coupling ensures a "more quantum" behavior of CT-MQC trajectories. $45-48|50| 67$

\section{Analysis of the correlation between Tors and HOOP}

The comparison of the dynamics generated by the two-dimensional Hahn-Stock model potentials $^{56}$ and by our three-dimensional model,,$\sqrt[27]{ }$ discussed in Section 2, highlighted the qualitative changes induced by the HOOP coordinate in the relaxation from $S_{1}$ to $S_{0}$ from cis-PSB3 to trans-PSB3. In order to investigate this effect further, in the following analysis, we will

- select representative CT-MQC trajectories to define the idea of "correlation" between motion along HOOP and along Tors for the three-dimensional model;

- point out the qualitative similar tendency of the trajectories, across methods, i.e., CTMQC and TSH-EDC, and across systems, i.e., PSB3 and a simplified rPSB11 in Rh, to successfully yield the trans- $S_{0}$ conformation mainly when the "correct" combination of Tors and HOOP velocities is achieved.

Last observation suggests that a two-state three-dimensional model is able to capture qualitatively the correlation between Tors and HOOP modes seen in simulations where the full set of degrees of freedom are considered. However, only certain basic topographic features of the PSB3 three-dimensional model are found in the PESs of rPSB11 in Rh. Common features are: (i) the initial $S_{1}$ gradient mainly directed along the BLA coordinate and only 
partially along the HOOP coordinate, (ii) the existence of a barrierless isomerization path and a nearly peaked minimum-energy CI point along the intersection space. On the other hand, as also mentioned above, the three-dimensional model features a force field that is symmetric with respect to both Tors and HOOP. This symmetry is completely lost in Rh. The consequences of such strong asymmetry is that in $\mathrm{Rh}$ the trajectories all relax in a counterclockwise direction rather than splitting in two equal populations relaxing in the clockwise and counterclockwise direction with identical probabilities.
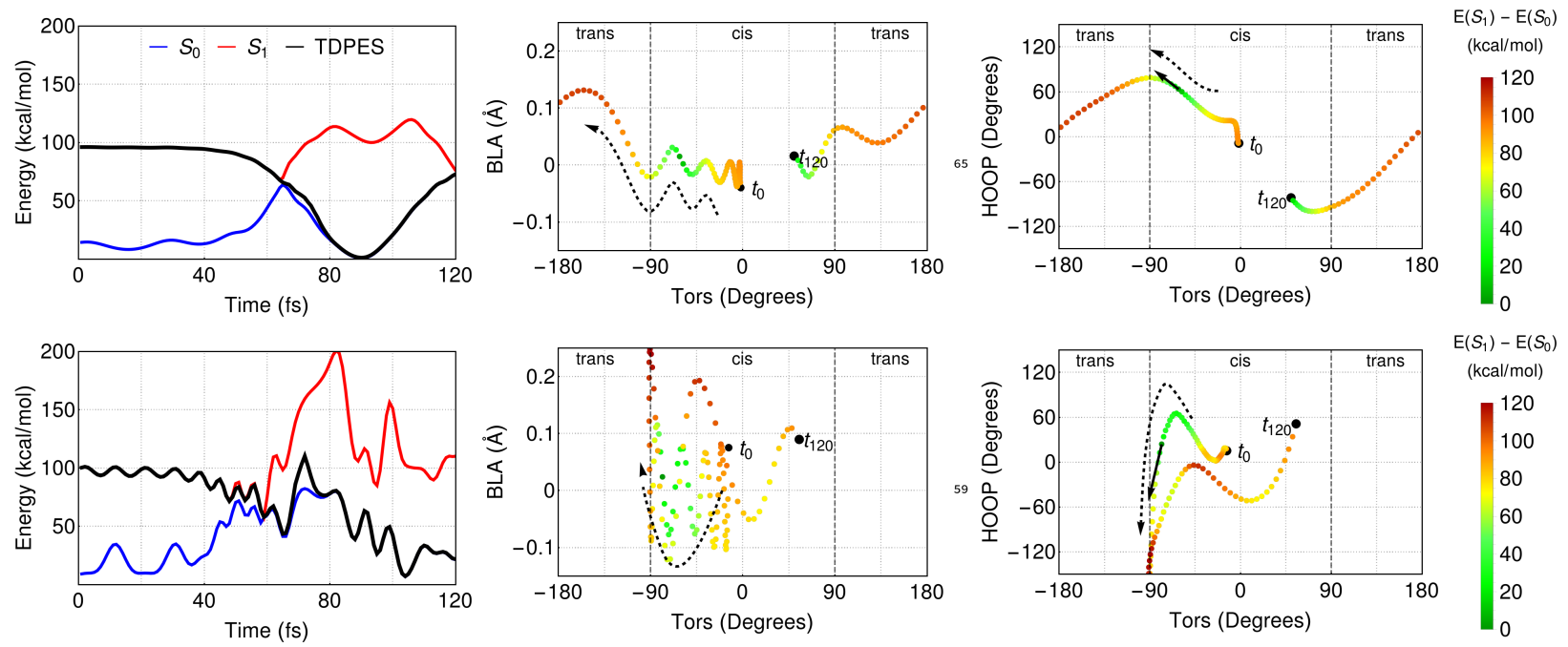

Figure 9: Left panels: profiles of the adiabatic $S_{0}$ (blue lines) and $S_{1}$ (red lines) potentials as functions of time, along two selected trajectories. The black curve is the TDPES of the CT-MQC method. Central panels: trajectories in the reduced Tors-BLA space starting at the point indicated with $t_{0}$ and ending after $120 \mathrm{fs}$ at the point $t_{120}$. Dashed vertical lines separate the cis from the trans conformations. Colors indicate the absolute value of the energy gap between $S_{1}$ and $S_{0}$ along the trajectories. Right panels: trajectories in the reduced Tors-HOOP space, with the straight arrows indicating the velocity at the point where the $S_{1} / S_{0}$ energy gap is minimum. In the central and right panels, the dashed arrows indicate the direction of time.

Figure 9 reports the potential energy profiles (left panels) along the CT-MQC dynamics of two selected representative trajectories together with their corresponding paths up to $120 \mathrm{fs}$. The trajectory geometrical paths are reported in the reduced Tors-BLA (central panels) and Tors-HOOP (right panels) space. Note that a single trajectory is not physically meaningful, especially in a coupled-trajectory scheme like CT-MQC. However, in our simulations we 
have identified a number of trajectories close to the two selected examples, and we will thus describe their behavior in detail. In CT-MQC, the potential energy driving the nuclear dynamics is the time-dependent potential energy surface (TDPES), which is basically the electronic Hamiltonian averaged over the instantaneous electronic state. The TDPES for the selected trajectories are compared in Fig. 9 (left panels) with the corresponding $S_{0}$ and $S_{1}$ profiles. Note that in the upper panel the trajectory shows a steep descent from $S_{1}$ to $S_{0}$ around $65 \mathrm{fs}$, and ends in a region of configuration space where $S_{0}$ decreases while $S_{1}$ increases with time. Conversely, in the lower panel, after switching from $S_{1}$ to $S_{0}$, the trajectory moves in a region where $S_{0}$ and $S_{1}$ have similar slopes (notice the in-phase oscillations of $S_{0}$ and $S_{1}$ between $60 \mathrm{fs}$ and $100 \mathrm{fs}$ ). Clearly, trajectories visiting different portion of the PESs have different behaviors, as it is shown in Fig. 9 (central panels). The trajectory depicted in the upper panel oscillates along BLA but propagates along Tors towards the trans-PSB3 product with values of Tors of $-180^{\circ}$, reaching the $S_{0}$ product in about $100 \mathrm{fs}$. The trajectory shown in the lower panel, instead, does not move towards the product, but it goes back to cis-PSB3 after a first passage through the CI region.

To understand the different reactive and non-reactive behaviors described above, we show the same two trajectories in the Tors-HOOP reduced configuration space (right panels). The velocity of the reactive trajectory (upper panel) at the hop point is directed towards increasing values of HOOP (positive HOOP velocity) and towards the photo-product (negative Tors velocity), as indicated by the straight arrow at the crossing point, whereas the velocity of the non-reactive trajectory (lower panel) at the crossing point has the opposite direction along HOOP (the Tors component still points towards trans-PSB3), as again indicated by the arrow. This analysis points to the existence of a phase relationship between Tors and HOOP velocities whose signs at the hop point, i.e., $S_{1}$ decay, determine the success of the isomerization. Such relationship, which has been previously documented using atomistic PSB3 ${ }^{31}$ and Rh models ${ }^{\sqrt{78168}}$ and probed experimentally ${ }^{17}$ implies that reactive trajectories are associated with negative (positive) Tors velocities and with positive (negative) HOOP 
velocities.
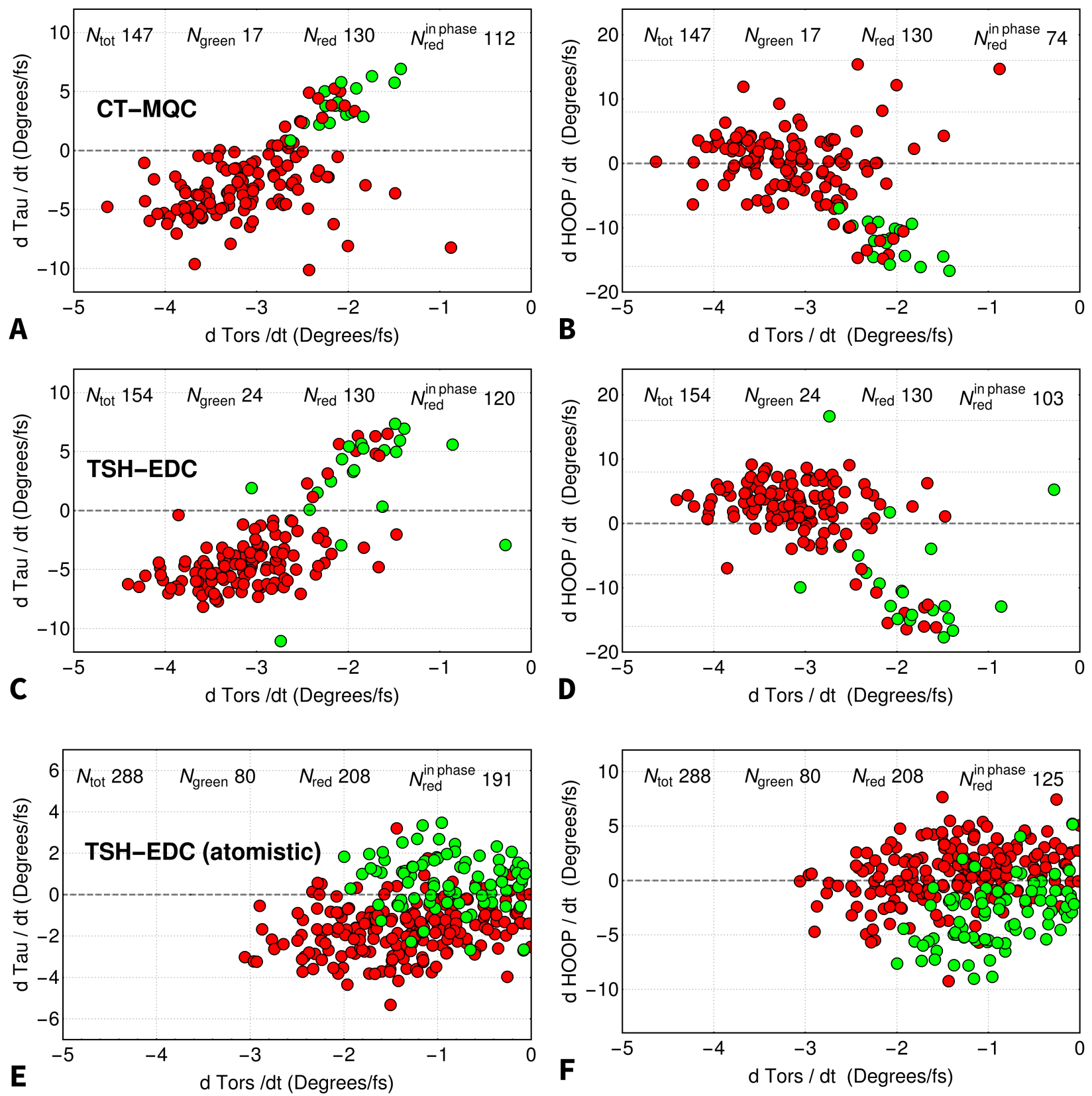

Figure 10: Panel A: Velocity of the overlap coordinate Tau vs. Tors velocities from CT-MQC. Panel B: Velocity of HOOP vs. Tors velocities from CT-MQC. The velocities are computed at the "hopping" time/position. Panels C-D: Same as in A-B, but for TSH-EDC. Panels E-F: Same as in A-B, but the results are extracted from TSH-EDC-based atomistic simulations of bovine $\mathrm{Rh}$ model with a simplified rPSB11 chromophore. ${ }^{7}$ Red dots refer to reactive trajectories, whereas green dots refer to trajectories that go back to the cis conformation.

In order to present results that can be directly compared with atomistic simulations found 
in the literature, ${ }^{17}$ in Fig. 10 we plot the velocity of the variable Tau $=$ Tors - HOOP $/ 2$ (panel A) and of the variable HOOP (panel B) against the velocity of Tors based on CT-MQC. We recall here that Tau stands for the overlap of the $\pi$-system orthogonal to the reactive double bond. The analysis is restricted to short-time dynamics after the first trajectory"hop" from $S_{1}$ to $S_{0}$. We designate that a CT-MQC trajectory switches from $S_{1}$ to $S_{0}$ if (i) it enters a region where the energy gap between $S_{1}$ and $S_{0}$ is less than $10 \mathrm{kcal} / \mathrm{mol} 4$. and (ii) for at least 3 consecutive time steps the TDPES is closer to $S_{0}$ than to $S_{1}$. The identification of these conditions becomes necessary because, as stated above, CT-MQC trajectories do not hop from one PESs to the other, but follow the TDPES. The velocities reported in Fig. 10 are computed at the hop point, and only the trajectories evolving from Tors $=0$ towards Tors $=-180^{\circ}$ are analyzed for consistency with the results of Fig. 9 . After the hop, that takes place in the vicinity of Tors $=-90^{\circ}$, as soon as a trajectory enters a region where Tors $\geq-30^{\circ}$ it becomes a non-reactive trajectory because it goes back to the cis geometry, whereas as soon as it enters a region where Tors $\leq-150^{\circ}$ it is identified as a reactive trajectory because it continues the isomerization process towards the trans geometry. Figure 10 (panels A-B) shows that over 147 analyzed trajectories, 130 are reactive (red dots) and 17 are non-reactive (green dots) suggesting a large quantum efficiency of about 0.88 (in agreement with the quantum yield estimated in Fig. 7 for CT-MQC in the time interval $50-100 \mathrm{fs}$ ). In panel A of Fig. 10 we show that all non-reactive trajectories have a positive value of the $\pi$-system overlap, i.e., Tau, velocity and a negative value of the Tors velocity; for 112 reactive trajectories, the velocities of the overlap and Tors have the same sign. Note that the variable HOOP enters with minus sign in the definition of Tau. All non-reactive trajectories have a negative HOOP velocity, as shown in panel B of Fig. 10 . Here, the dominating role of the HOOP velocity in directing the trajectories towards the product conformation is displayed. Again, the described interplay between Tors and HOOP just analyzed confirms a mechanism for the $S_{1}$ to $S_{0}$ population decay well beyond the usually

\footnotetext{
${ }^{4}$ Analyses with the values 5 and $7 \mathrm{kcal} / \mathrm{mol}$ have been performed as well, but the considerations remain the same.
} 
adopted one-dimensional Landau-Zener model,,$\frac{10}{10}$ despite the fact that the large energy gap between the electronic states in PSB3 (of the order of $4 \mathrm{eV}$, thus about $1.5 \mathrm{eV}$ larger than the one of $\mathrm{Rh}$ ) gives rise to very steep slopes towards peaked CIs along which one would expect the trajectories to display a one-dimensional-like dynamics.

To put our observations in a broader perspective, and validate the results provided by CT-MQC, we conclude this analysis including the model-based results (panels C-D) and the atomistic results (panels E-F) using TSH-EDC. This last set of results has been extracted from Ref ${ }^{7}$ where a QM/MM study of a simplified rPSB11 inserted in the protein cavity has been reported. The pattern in panels A-B is similar to those in panels C-D and E-F, even though in the latter a lower isomerization quantum efficiency can be extracted (of 0.72) if compared to panels A-B and panels C-D (quantum efficiency of 0.84). Qualitatively, CTMQC and TSH-EDC estimate of the quantum efficiency from Fig. 10 agrees with the trend shown in Fig. 7just after $80-100$ fs.

These final observations, together with the early 50 fs decay and vibrational coherence seen above, strongly support the interest in developing model PESs allowing for in-depth analysis of the origin of certain critical features of the cis-trans isomerization in rPSB11-like systems and of similarities/differences between quantum and quantum-classical schemes.

\section{Conclusions}

In the extensive analysis reported in this work on the photoisomerization of a retinal chromophore model, we have

- presented how to perform vibronic wavepacket dynamics driven by the curvilinear coordinates BLA, Tors and HOOP in Section 2;

- discussed the contribution of the HOOP mode on the dynamics (probabilities and nuclear kinetic energy) by comparing our three-dimensional model with the two-dimensional Hahn-Stock model $\sqrt{56}$ in Sections 2.1 and 2.2; 
- studied nuclear quantum dynamics and CT-MQC trajectories, and their relation with the topology of the adiabatic PESs in Section 3;

- pointed out differences and similarities between quantum and quantum-classical simulations, as well as among the studied quantum-classical methods themselves in Sections 3.1 and 3.2 ;

- identified qualitative analogies between CT-MQC and TSH-EDC, as well as between PSB3 and a rPSB11-like system in Rh, in capturing the correlation between Tors and HOOP velocities to yield the trans- $S_{0}$ conformation in Section 4.

In summary, recently parametrized analytical three-dimensional PESs ${ }^{27}$ have allowed to employ CT-MQC and other quantum-classical methods to study the isomerization of PSB3. The recently-developed CT-MQC scheme provided a description of the excited-state dynamics in slightly better agreement with quantum dynamics calculations with respect to other quantum-classical schemes. Based on our combined quantum and quantum-classical analysis, we validated some observations that have been previously reported in the literature

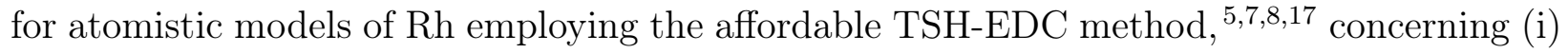
the three-dimensional nature of the isomerization coordinate, (ii) the coherent vibrational motion, (iii) the correlation between HOOP and Tors velocities to reach the photo-products, and (iv) the nature of the non-adiabatic crossing.

When comparing the three-dimensional model PESs ${ }^{27}$ with a two-dimensional model, $\underline{56}$ we have shown the suppression of the efficient $S_{1}$ decay within the 200 fs simulated dynamics. This observation might indicate the critical role played by additional intramolecular degrees of freedom in reaching and stabilizing the photo-products. Instead, the similarities between dynamics based on the three-dimensional model $\mathrm{PESs}{ }^{27}$ and atomistic QM/MM results ${ }^{7}$ suggest that the correlation between HOOP (or Tau) and Tors velocities is a general, essential property to steer the isomerization of rPSB11-like systems towards the photo-product formation independently on the environment. 
To reveal quantum effects, we have compared vibronic wavepacket dynamics and trajectorybased schemes, only accessible in reduced-dimensionality PES models. In particular, at short times after the photo-excitation the trans conformer in $S_{0}$ is predicted to be produced with higher probability, i.e., with a higher quantum efficiency, when using quantum-classical trajectory propagations rather than quantum dynamics. This behavior appears to be the consequence of a too fast funneling process through the CIs leading the trajectories to acquire a large amount of kinetic energy along the low-frequency Tors mode, with corresponding loss of potential energy, BLA and HOOP kinetic energy.

TSH-EDC is widely used in atomistic simulations, thus the disagreement with respect to quantum dynamics discussed in this work might suggest that TSH-EDC is not fully adequate to describe the photo-induced isomerization in rPSB11-like systems. However, we expect that inclusion of additional degrees of freedom and/or an environment might be the key to induce a more classical-like behavior that is better captured by the trajectory-based scheme. Another feature that indeed plays a role in the formation of the photo-products is the broken symmetry and augmented slope along Tors of the PESs acting on the rPSB11 chromophore of $\mathrm{Rh}$, due to the chiral protein environment hosting the chromophore, that steers the isomerization exclusively in the counterclockwise direction. The effect of both factors will be the focus of future studies on the dynamics of PSB3.

An important aspect of the photo-isomerization process that has not been addressed in this work is the effect of natural, incoherent light excitation. Natural light excitation prepares the system in a mixture of eigenstates of the total Hamiltonian, whose time evolution is expected to proceed incoherently. Therefore, the predicted quantum yield is potentially affected by such an incoherent dynamics. Thanks to developments of theoretical approaches able to account for incoherent light excitation adopting quantum $2455 / 69$ (71 and quantumclassical ${ }^{[72}$ perspectives, future work in this direction on the three-dimensional model might provide valuable information. 


\section{Acknowledgement}

Xuchun Yang is gratefully acknowledged for providing the atomistic data. E. M., D. L., F. A. thank CNRS for financial support via the International Emerging Action. M.O. acknowledges partial funding from grant NSF CHE-CLP-1710191 and MIUR PRIN 2015.

\section{Supporting Information Available}

The following files are available free of charge.

- Dynamics_Adia_Tors_BLA.mp4: it contains an animation showing the quantummechanical evolution of the Tors-BLA reduced densities in $S_{0}$ and in $S_{1}$ along with classical dynamics based on the CT-MQC scheme up to 200 fs.

- Dynamics_Adia_Tors_HOOP.mp4: it contains an animation showing the quantummechanical evolution of the Tors-HOOP reduced densities in $S_{0}$ and in $S_{1}$ along with classical dynamics based on the CT-MQC scheme up to $200 \mathrm{fs}$.

\section{A. Analytical model potentials}

The electronic Hamiltonian in the diabatic basis including the two lowest states of PSB3 as functions of the three vibrational modes Tors, $\mathrm{BLA}, \mathrm{HOOP}=\theta, r, \phi$ is

$$
\hat{H}_{e l}(\theta, r, \phi)=\left(\begin{array}{cc}
H_{c o v}(\theta, r, \phi) & H_{c p}(\theta, r, \phi) \\
H_{c p}(\theta, r, \phi) & H_{c t}(\theta, r, \phi) .
\end{array}\right)
$$

The expressions we used for $H_{c o v}(\theta, r, \phi)$ and $H_{c t}(\theta, r, \phi)$ are similar to those in Ref., ${ }^{27}$ namely

$$
\begin{gathered}
H_{c o v}(\theta, r, \phi)=H_{\operatorname{cov} 2 D}(\theta, r, \phi)+H_{\text {corr }}(\phi) \\
H_{c t}(\theta, r, \phi)=H_{c t 2 D}(\theta, r, \phi)+H_{c o r r}(\phi)
\end{gathered}
$$


with $H_{c t 2 D}(\theta, r, \phi)$ having the same expression as in Ref. ${ }^{27}$ In this work, we have slightly modified $H_{\text {corr }}(\phi), H_{\operatorname{cov} 2 D}(\theta, r, \phi)$ and $H_{c p}(\theta, r, \phi)$, and the new expressions, with angle variables indicated in radians, are

$$
\begin{gathered}
H_{\text {corr }}(\phi)=h_{1} \sin ^{2}\left(\frac{\phi}{4}\right) \\
H_{\text {cov } 2 D}(\theta, r, \phi)=\left(V_{\text {Morse }_{1}}(r)+d_{2}\right) \sin ^{2}\left(\theta-\frac{\phi}{2}\right)+d_{3} \cos ^{2}\left(\frac{\theta}{2}-\frac{\phi}{4}\right)+V_{\text {Morse }_{2}}(r) \cos ^{2}\left(\theta-\frac{\phi}{2}\right)
\end{gathered}
$$

and

$$
H c p(\theta, r, \phi)=k_{1} \sin (2 \theta-\phi)
$$

Note that $H_{c p}(\theta, r, \phi)=H_{c p}(\theta, \phi)$ is independent of $r$. In Eq. (19) the Morse potential has replaced the harmonic potential of the original model, and we have

$$
\begin{aligned}
& V_{\text {Morse }_{1}}(r)=d_{r}\left(-1+e^{-d_{1}\left(r-r_{T S}\right)}\right)^{2} \\
& V_{\text {Morse }_{2}}(r)=d_{r}\left(-1+e^{-d_{4}\left(r-r_{\text {min }}\right)}\right)^{2}
\end{aligned}
$$

with $d_{1}=\sqrt{k f_{1} /\left(2 d_{r}\right)}$ and $d_{4}=\sqrt{k f_{4} /\left(2 d_{r}\right)}$. The parameter $k f_{1}$ is the BLA force constant evaluated at the position of the covalent character transition state $\left(T S_{C O V}\right)$, whereas $k f_{4}$ is the BLA force constant evaluated for the cis or the trans isomers (the force constants are considered the same for both isomers). Finally, the depth well, $d_{r}$ as been fixed at a value of $2000 \mathrm{kcal} . \mathrm{mol}^{-1}$, while $r_{T S}=0.02508 \AA$ and $r_{\min }=0.09126 \AA$ are the optimal BLA values at the $T S_{C O V}$ for cis and trans geometries, respectively. Here, we assume, that the optimal BLA values are the same for the both cis and trans isomers. Table 2 indicates the values of the parameters used in the new model potentials. The modifications just described 
reduce the number of parameters, and improve the agreement of the model when compared with the dataset used for the fitting. The model potentials used in this work along with the original potentials of Ref., 27 the Hahn-Stock potentials and the constant metric tensor used in Section 2 are available for download. $\frac{73}{73}$

Table 2: Potential parameters.

\begin{tabular}{cc|cc|cc}
$H_{\text {cov } 2 D}$ & & $H_{\text {corr }}$ & $H_{c p}$ & \\
\hline \hline$k f_{1}$ & $3733 \mathrm{kcal} . \mathrm{mol}^{-1} . \AA^{-2}$ & $h_{1}$ & $155.7 \mathrm{kcal} . \mathrm{mol}^{-1}$ & $k_{1}$ & $24.04 \mathrm{kcal}_{\mathrm{mol}} \mathrm{mol}^{-1}$ \\
$d_{2}$ & $54.63 \mathrm{kcal} . \mathrm{mol}^{-1}$ & & & & \\
$d_{3}$ & $3.801 \mathrm{kcal} . \mathrm{mol}^{-1}$ & & & & \\
$k f_{4}$ & $1097 \mathrm{kcal} . \mathrm{mol}^{-1} . \AA^{-2}$ & & & & \\
$d_{r}$ & $2000 \mathrm{kcal} . \mathrm{mol}^{-1}$ & & & & \\
$r_{T S}$ & $0.02508 \AA$ & & & & \\
$r_{\text {min }}$ & $0.09126 \AA$ & & &
\end{tabular}

\section{B. Kinetic energy Operator}

For a molecular system composed by $N$ atoms, the Kinetic Energy Operator (KEO) in terms of $n$ curvilinear coordinates $\mathbf{Q}=\left\{Q_{1} \ldots Q_{n}\right\}$, can be expressed as $5960 \mid 74$

$$
\hat{\mathcal{K}}=\frac{1}{2} \sqrt{\frac{J(\mathbf{Q})}{\rho(\mathbf{Q})}} \sum_{i, j} J(\mathbf{Q})^{-1} \hat{P}_{i} J(\mathbf{Q}) G^{i j}(\mathbf{Q}) \hat{P}_{j} \sqrt{\frac{\rho(\mathbf{Q})}{J(\mathbf{Q})}}
$$

where: $\hat{P}_{i}$ 's are the conjugated momenta to the active coordinates including the three components, $\hat{J}_{\alpha}$, of the total angular momentum; $G^{i j}(\mathbf{Q})$ are the contravariant components of the metric tensor; $J(\mathbf{Q})$ is defined as $1 / \sqrt{\operatorname{det}(\mathbf{G}(\mathbf{Q}))}$ and is the Jacobian of the coordinate transformation when the molecular system is unconstrained $(n=3 N-6)$; $\rho(\mathbf{Q})$ is the normalization weight associated to the deformation part of the volume element $d \tau_{d e f}=\rho(\mathbf{Q}) \cdot d Q_{1} \cdot d Q_{2} \cdots d Q_{n}$ and it means that a non-Euclidean normalization convention is used $(\rho(\mathbf{Q}) \neq J(\mathbf{Q}))$.

There exist several procedures to derive the expression of the metric tensor. Usually, for the numerical approach, as in TNUM, ${ }^{58}$ the so-called $\mathbf{x}(\mathbf{Q})$ approach $^{[77}$ is used for which the 
covariant components of the metric tensor, $g_{i j}(\mathbf{Q})$, are obtained first, then the contravariant components, $G^{i j}(\mathbf{Q})$, are calculated. The corresponding matrix $\mathbf{G}$ is expressed as

$$
\mathbf{G}(\mathbf{Q})=\mathbf{g}(\mathbf{Q})^{-1}=\left(\begin{array}{cc}
\mathbf{S}(\mathbf{Q}) & \mathbf{C}^{t}(\mathbf{Q}) \\
\mathbf{C}(\mathbf{Q}) & \mathbf{I}(\mathbf{Q})
\end{array}\right)^{-1}
$$

where, $\mathbf{S}(\mathbf{Q}), \mathbf{C}(\mathbf{Q})$ and $\mathbf{I}(\mathbf{Q})$ are, respectively, the deformation contribution, the Coriolis contribution, and the inertia tensor. Their expressions are

$$
\begin{aligned}
S_{i j}(\mathbf{Q}) & =\sum_{k} m_{k} \frac{\partial \mathbf{R}_{k}}{\partial Q_{i}} \cdot \frac{\partial \mathbf{R}_{k}}{\partial Q_{j}} \\
C_{\alpha j}(\mathbf{Q}) & =\sum_{k} m_{k}\left[\mathbf{R}_{k} \times \frac{\partial \mathbf{R}_{k}}{\partial Q_{j}}\right]_{\alpha} \\
I_{\alpha \beta}(\mathbf{Q}) & =\sum_{k} m_{k}\left(\mathbf{R}_{k} \cdot \mathbf{R}_{k}\right) \delta_{\alpha \beta}-\left[\mathbf{R}_{k}\right]_{\alpha}\left[\mathbf{R}_{k}\right]_{\beta}
\end{aligned}
$$

where $\mathbf{R}_{k}$ and $m_{k}$ are, respectively, the body fixed Cartesian vector positions and the mass of atom $k$. Furthermore, the $\alpha$-th component $(\alpha=\{x, y, z\})$ of a vector $\mathbf{X}$ is denoted $[\mathbf{X}]_{\alpha}$.

Equation (23) can be transformed to give Eq. (4) for the deformation part of the KEO. 58160 Numerically, the functions, $f_{2}^{i j}(\mathbf{Q}), f_{1}^{i}(\mathbf{Q})$ and $V_{e p}(\mathbf{Q})$, can be obtained exactly to working precision resorting to automatic differentiation. $\underline{58 / 78}$

\section{Computational details}

The initial wavepacket is represented as product of uni-dimensional Gaussians. More specifically, for the $i$-th coordinate $Q^{i}$, the unnormalized expression of the Gaussian is given by $\exp \left[-\left(Q^{i}-Q_{0}^{i}\right)^{2} / \Delta Q^{i^{2}}\right]$, where $Q_{0}^{i}$ is the value of $Q^{i}$ at the cis geometry and $\Delta Q^{i}$ is width of the Gaussian. $\Delta Q^{i}$ is computed as $\sqrt{2} /\left(\sqrt[4]{k_{i} / G_{c i s}^{i i}}\right)$ where $k_{i}$ and $G_{c i s}^{i i}$ are the force constant and the metric tensor component at the photo-reactant geometry, respectively.

For the quantum-dynamics simulations, the nuclear wavepacket has been expanded in a 
Table 3: Parameters in atomic units used for the initial wavepacket: force constants, metric tensor's contravariant components, width $\Delta Q^{i}$, and center $Q_{0}^{i}$ of the initial Gaussian.

\begin{tabular}{cc|cccc} 
Force constant & & $\mathbf{G}_{c i s}$ diagonal values & & $\Delta Q^{i}$ & $Q_{0}^{i}$ \\
\hline \hline$k_{r}$ & 0.4896 & $G_{c i s}^{r r} \equiv 1 / M_{r}$ & $6.965 \cdot 10^{-5}$ & 0.1544 & 0.1725 \\
$k_{\theta}$ & 0.1198 & $G_{c i s}^{\theta \theta} \equiv 1 / I_{\theta}$ & $3.381 \cdot 10^{-5}$ & 0.1833 & 0.0 \\
$k_{\phi}$ & 0.06098 & $G_{c i s}^{\phi \phi} \equiv 1 / I_{\phi}$ & $41.48 \cdot 10^{-5}$ & 0.4061 & 0.0
\end{tabular}

3D-basis set formed by the direct-product of three uni-dimensional basis sets for each diabatic state. The harmonic-oscillator (HO) basis set has been used for BLA and HOOP, while Fourier-series periodic basis set has been used for Tors. The consistency of the grid point and the basis function numbers has been tested increasing, progressively, the basis set sizes until convergence of propagation was reached.

Table 4: Parameters in atomic units of the uni-dimensional basis sets for each coordinate, $Q^{i}$. $n b$ and $n q$ are, respectively, the numbers of basis functions and grid points. The HO basis functions are expressed as: $H_{m}\left[S c^{i}\left(Q^{i}-Q_{0}^{i}\right)\right] \cdot \exp \left[-1 / 2 \cdot\left(S c^{i}\left(Q^{i}-Q_{0}^{i}\right)\right)^{2}\right]$ where, $H_{m}$ is the normalized $m$-th Hermite polynomial, $S c^{i}\left(=\sqrt[4]{k_{i} / G_{c i s}^{i i}}\right)$ is a scaling parameter and $Q_{0}^{i}$ is the center of basis set.

\begin{tabular}{c|c|cc|cc}
$Q^{i}$ & type & $n b$ & $n q$ & $Q_{0}^{i}$ & $S c^{i}$ \\
\hline \hline$r$ & HO & 20 & 32 & 0.1725 & 9.157 \\
$\theta$ & Fourier & 128 & 136 & & \\
$\phi$ & HO & 24 & 60 & 0.0 & 3.482
\end{tabular}

The propagation was performed with the Chebychev scheme for which the evolution operator is expanded on Chebychev polynomials. $\frac{79}{79}$ The Hamiltonian needs to be renormalized so that its spectral range lies between $[-1,1]$. Finally, the wavepacket has been propagated for 200 fs using a time step of 1 fs with the initial wavepacket starting on $S_{1}$.

The used trajectory-based methods are: the Ehrenfest scheme, $\stackrel{42}{t}$ the CT-MQC approach $\frac{47 / 48}{}$ derived from the exact factorization of the electron-nuclear wavefunction, fewestswitches Tully surface hopping (TSH), 43 including its version with energy decoherence corrections (TSH-EDC) $\stackrel{44}{4}$ For all methods, the bundle of trajectories at the initial time has been generated from a (harmonic) Wigner distribution determined from the initial quantum 
wavepacket. The variances $\left(\Delta Q_{i}^{2} / \sqrt{2}\right)$ of the initial Gaussians can be determined from the values of the force constants and the masses (or of the elements of the metric tensor) given in Table 3 .

Quantum-classical simulations are run in the adiabatic basis. The adiabatic PESs are determined by diagonalizing the Hamiltonian (15). The non-adiabatic couplings have been computed analytically with the usual Hellmann-Feynman theorem $\frac{80}{82}$ from the eigenvectors of the Hamiltonian (15). This procedure is implemented for all potentials in the ModelLib library, ${ }^{73}$ and in particular for the three-dimensional PSB3 model.

A set of $N_{t r a j}=600$ initial conditions have been generated and used for all quantumclassical methods. For TSH and TSH-EDC calculations, each initial condition has been used to generate 10 trajectories by varying the random seed for the stochastic jumps, yielding a total of 6000 trajectories.

All simulations have been carried out for 200 fs with a time step of 0.0025 fs. Periodic boundary conditions are used for Tors and HOOP, with periodicity $2 \pi$ and $4 \pi$, respectively.

In CT-MQC simulations, the effect of the quantum momentum has been switched off when the trajectories are completely distributed all over the available configuration space, namely at 120 fs. The code used for quantum-classical simulations is a modified, developer version of the CTMQC code. .83

\section{References}

(1) Ernst, O. P.; Lodowski, D. T.; Elstner, M.; Hegemann, P.; Brown, L. S.; Kandori, H. Microbial and animal rhodopsins: Structures, functions, and molecular mechanisms. Chem. Rev. 2014, 114, 126-163.

(2) Schoenlein, R. W.; Peteanu, L. A.; Mathies, R. A.; Shank, C. V. The first step in vision: Femtosecond isomerization of rhodopsin. Science 1991, 254, 412-415.

(3) Wang, Q.; Schoenlein, R. W.; Peteanu, L. A.; Mathies, R. A.; Shank, C. V. Vibrationally 
Coherent Photochemistry in the Femtosecond Primary Event of Vision. Science 1994, 266, 422-424.

(4) Kukura, P.; McCamant, D. W.; Yoon, S.; Wandschneider, D. B.; Mathies, R. A. Structural Observation of the Primary Isomerization in Vision with Femtosecond-Stimulated Raman. Science 2005, 310, 1006-1009.

(5) Polli, D.; Altoè, P.; Weingart, O.; Spillane, K. M.; Manzoni, C.; Brida, D.; Tomasello, G.; Orlandi, G.; Kukura, P.; Mathies, R. A.; Garavelli, M.; Cerullo, G. Conical intersection dynamics of the primary photoisomerization event in vision. $\mathrm{Na}$ ture 2010, 467, 440-445.

(6) Johnson, P. J. M.; Halpin, A.; Morizumi, T.; Ernst, V. I. P. O. P.; Miller, R. J. D. Local vibrational coherences drive the primary photochemistry of vision. Nature Chem. 2015, 7, 980-986.

(7) Schnedermann, C.; Yang, X.; Liebel, M.; Spillane, K. M.; Lugtenburg, J.; Fernández, I.; Valentini, A.; Schapiro, I.; Olivucci, M.; Kukura, P.; Mathies, R. A. Evidence for a vibrational phase-dependent isotope effect on the photochemistry of vision. Nature Chem. 2018, 10, 449-455.

(8) Schapiro, I.; Ryazantsev, M. N.; Frutos, L. M.; Ferré, N.; Lindh, R.; Olivucci, M. The Ultrafast Photoisomerizations of Rhodopsin and Bathorhodopsin are Modulated by Bond Alternation and HOOP driven Electronic Effects. J. Am. Chem. Soc. 2011, 133, 3354-3364.

(9) Manathunga, M.; Yang, X.; Orozco-Gonzalez, Y.; Olivucci, M. Impact of Electronic State Mixing on the Photoisomerization Time Scale of the Retinal Chromophore. J. Phys. Chem. Lett. 2017, 8, 5222-5227.

(10) El-Tahawy, M. M. T.; Nenov, A.; Weingart, O.; Olivucci, M.; Garavelli, M. Relationship between Excited State Lifetime and Isomerization Quantum Yield in Animal 
Rhodopsins: Beyond the One-Dimensional Landau-Zener Model. J. Phys. Chem. Lett. 2018, 9, 3315-3322.

(11) Filatov, M.; Min, S. K.; Kim, K. S. Direct Nonadiabatic Dynamics by Mixed QuantumClassical Formalism Connected with Ensemble Density Functional Theory Method: Application to trans-Penta-2,4-dieniminium Cation. J. Chem. Theory Comput. 2018, 14, 4499-4512.

(12) Schapiro, I.; Gueye, M.; Paolino, M.; Fusi, S.; Marchand, G.; Haacke, S.; Martin, M. E.; Huntress, M.; Vysotskiy, V. P.; Léonard, V. V.; Olivucci, M. Synthesis, spectroscopy and QM/MM simulations of a biomimetic ultrafast light-driven molecular motor. Photochem. Photobiol. Sci. 2019, 18, 2259-2269.

(13) González-Luque, R.; Garavelli, M.; Bernardi, F.; Merchán, M.; Robb, M. A.; Olivucci, M. Computational evidence in favor of a two-state, two-mode model of the retinal chromophore photoisomerization. Proc. Nat. Ac. Sci. 2000, 17, 9379-9384.

(14) Ferré, N.; Olivucci, M. Probing the Rhodopsin Cavity with Reduced Retinal Models at the CASPT2//CASSCF/AMBER Level of Theory. J. Am. Chem. Soc. 2003, 125, 6868-6869.

(15) Andruniów, T.; Ferré, N.; Olivucci, M. Structure, initial excited-state relaxation, and energy storage of rhodopsin resolved at the multiconfigurational perturbation theory level. Proc. Natl. Acad. Sci. U. S. A. 2004, 101, 17908-17913.

(16) Coto, P. B.; Strambi, A.; Ferré, N.; Olivucci, M. The color of rhodopsins at the ab initio multiconfigurational perturbation theory resolution. Proc. Natl. Acad. Sci. U. S. A. 2006, 103, 17154-17159.

(17) Frutos, L. M.; Andruniów, T.; Santoro, F.; Ferré, N.; Olivucci, M. Tracking the Excitedstate Time Evolution of the Visual Pigment with Multiconfigurational Quantum Chemistry. Proc. Natl. Acad. Sci. U. S. A. 2007, 104, 7764-7769. 
(18) Gozem, S.; Luk, H. L.; Schapiro, I.; Olivucci, M. Theory and Simulation of the Ultrafast Double-Bond Isomerization of Biological Chromophores. Chem. Rev. 2017, 117, 1350213565.

(19) Virshup, A. M.; Punwong, C.; Pogorelov, T. V.; Lindquist, B. A.; Ko, C.; Martínez, T. J. Photodynamics in Complex Environments: Ab Initio Multiple Spawning Quantum Mechanical/Molecular Mechanical Dynamics. J. Phys. Chem. B 2009, 113, 3280-3291.

(20) Punwong, C.; Martínez, T. J.; Hannongbua, S. Direct QM/ MM Simulation of Photoexcitation Dynamics in Bacteriorhodopsin and Halorhodopsin. Chem. Phys. Lett. 2014, 213-218, 610-611.

(21) Seidner, L.; Domcke, W. Microscopic modelling of the photoisomerization and internalconversion dynamics. Chem. Phys. 1994, 186, 27-40.

(22) Hahn, S.; Stock, G. Femtosecond secondary emission arising from the nonadiabatic photoisomerization in rhodopsin. Chem. Phys. 2000, 259, 297-312.

(23) Olivucci, M.; Lami, A.; Santoro, F. A Tiny Excited-State Barrier Can Induce a Multiexponential Decay of the Retinal Chromophore: A Quantum Dynamics Investigation. Ang. Chemie Int. Ed. 2005, 44, 5118-5121.

(24) Tscherbul, T. V.; Brumer, P. Quantum coherence effects in natural light-induced processes: cis-trans photoisomerization of model retinal under incoherent excitation. Phys. Chem. Chem. Phys. 2015, 17, 30904-30913.

(25) Farag, M. H.; Jansen, T. L. C.; Knoester, J. Probing the Interstate Coupling near a Conical Intersection by Optical Spectroscopy. J. Phys. Chem. Lett. 2016, 7, 3328-3334.

(26) Farag, M. H.; Jansen, T. L. C.; Knoester, J. The origin of absorptive features in the two-dimensional electronic spectra of rhodopsin. Phys. Chem. Chem. Phys. 2018, 20, $12746-12754$. 
(27) Marsili, E.; Farag, M. H.; Yang, X.; Vico, L. D.; Olivucci, M. Two-State, Three-Mode Parametrization of the Force Field of a Retinal Chromophore Model. J. Phys. Chem. A 2019, 123, 1710-1719.

(28) Garavelli, M.; Celani, P.; Bernardi, F.; Robb, M. A.; Olivucci, M. The C5H6NH2+ Protonated Schiff Base: An ab Initio Minimal Model for Retinal Photoisomerization. J. Am. Chem. Soc. 1997, 119, 6891-6901.

(29) Vreven, T.; Bernardi, F.; Garavelli, M.; Olivucci, M.; Robb, M. A.; Schlegel, H. B. Ab Initio Photoisomerization Dynamics of a Simple Retinal Chromophore Model. J. Am. Chem. Soc. 1997, 119, 12687-12688.

(30) Weingart, O.; Migani, A.; Olivucci, M.; Robb, M. A.; Buss, V.; Hunt, P. Probing the Photochemical Funnel of a Retinal Chromophore Model via Zero-Point Energy Sampling Semiclassical Dynamics. J. Phys. Chem. A 2004, 108, 4685-4693.

(31) Weingart, O. The role of HOOP-modes in the ultrafast photo-isomerization of retinal models. Chem. Phys. 2008, 349, 348-355.

(32) Barbatti, M.; Ruckenbauer, M.; Szymczak, J. J.; Aquino, A. J. A.; Lischka, H. Nonadiabatic excited-state dynamics of polar p-systems and related model compounds of biological relevance. Phys. Chem. Chem. Phys. 2008, 10, 482-494.

(33) Gozem, S.; Melaccio, F.; Valentini, A.; Filatov, M.; Huix-Rotllant, M.; Ferré, N.; Frutos, L. M.; Angeli, C.; Krylov, A. I.; Granovsky, A. A.; Lindh, R.; Olivucci, M. Shape of multireference, equation-of-motion coupled-cluster, and density functional theory potential energy surfaces at a conical intersection. J. Chem. Theory Comp. 2014, 10, 3074-3084.

(34) Klaffki, N.; Weingart, O.; Garavelli, M.; Spohr, E. Sampling excited state dynamics: influence of HOOP mode excitations in a retinal model. Phys. Chem. Chem. Phys. 2014, 14, 14299-14305. 
(35) Tuna, D.; Lefrancois, D.; Wolański, Ł.; Gozem, S.; Schapiro, I.; Andruniów, T.; Dreuw, A.; Olivucci, M. Assessment of Approximate Coupled-Cluster and AlgebraicDiagrammatic-Construction Methods for Ground- and Excited-State Reaction Paths and the Conical-Intersection Seam of a Retinal-Chromophore Model. J. Chem. Theory Comp. 2015, 11, 5758-5781.

(36) Gozem, S.; Huntress, M.; Schapiro, I.; andA. A. Granovsky, R. L.; Angeli, C.; Olivucci, M. Dynamic electron correlation effects on the ground state potential energy surface of a retinal chromophore model. J. Chem. Theory Comp. 2012, 8, 4069-4080.

(37) Gozem, S.; Melaccio, F.; R. Lindh, R.; Krylov, A. I.; Granovsky, A. A.; Angeli, C.; Olivucci, M. Mapping the excited state potential energy surface of a retinal chromophore model with multireference and EOM-CC methods. J. Chem. Theory Comput. 2013, 9, 4495-4506.

(38) Johnson, P. J. M.; Farag, M. H.; Halpin, A.; Morizumi, T.; Prokhorenko, V. I.; Knoester, J.; Jansen, T. L. C.; Ernst, O. P.; Miller, R. J. D. The Primary Photochemistry of Vision Occurs at the Molecular Speed Limit. J. Phys. Chem. B 2017, 121, 4040-4047.

(39) Granovsky, A. A. Extended multi-configuration quasi- degenerate perturbation theory: The new approach to multi-state multi-reference perturbation theory. J. Chem. Phys. 2011, 134, 214113-214127.

(40) Schnedermann, C.; Liebel, M.; Kukura, P. Mode-Specificity of Vibrationally Coherent Internal Conversion in Rhodopsin during the Primary Visual Event. J. Am. Chem. Soc. 2015, 137, 2886-2891.

(41) Lauvergnat, D. ElVibRot-TnumTana Quantum Dynamics Code. https://github. com/lauvergn/ElVibRot-TnumTana.

(42) Tully, J. C. Mixed quantum-classical dynamics. Faraday Discuss. 1998, 110, 407. 
(43) Tully, J. C. Molecular dynamics with electronic transitions. J. Chem. Phys. 1990, 93, 1061.

(44) Granucci, G.; Persico, M. Critical appraisal of the fewest switches algorithm for surface hopping. J. Chem. Phys. 2007, 126, 134114.

(45) Min, S. K.; Agostini, F.; U.Gross, E. K. Coupled-Trajectory Quantum-Classical Approach to Electronic Decoherence in Nonadiabatic Processes. Phys. Rev. Lett. 2015, 115,73001 .

(46) Agostini, F.; Min, S. K.; Abedi, A.; Gross, E. K. U. Quantum-Classical Nonadiabatic Dynamics: Coupled- vs Independent-Trajectory Methods. J. Chem. Theory Comput. 2016, 12, 2127-2143.

(47) Min, S. K.; Agostini, F.; Tavernelli, I.; Gross, E. K. U. Ab Initio Nonadiabatic Dynamics with Coupled Trajectories: A Rigorous Approach to Quantum (De)Coherence. J. Phys. Chem. Lett. 2017, 8, 3048-3055.

(48) Gossel, G. H.; Agostini, F.; Maitra, N. T. Coupled-Trajectory Mixed Quantum-Classical Algorithm: A Deconstruction. J. Chem. Theory Comput. 2018, 14, 4513-4529.

(49) Curchod, B. F. E.; Agostini, F.; Tavernelli, I. CT-MQC - A Coupled-Trajectory Mixed Quantum/Classical method including nonadiabatic quantum coherence effects. Euro. Phys. J. B 2018, 91, 168.

(50) Talotta, F.; Morisset, S.; Rougeau, N.; Lauvergnat, D.; Agostini, F. Spin-Orbit Interactions in Ultrafast Molecular Processes. Phys. Rev. Lett. 2020, 124, 033001.

(51) Abedi, A.; Maitra, N. T.; Gross, E. K. U. Exact Factorization of the Time-Dependent Electron-Nuclear Wave Function. Phys. Rev. Lett. 2010, 105, 123002.

(52) Agostini, F.; Curchod, B. F. E. Different flavors of nonadiabatic molecular dynamics. WIREs Comput. Mol. Sci. 2019, 9, e1417. 
(53) Abe, M.; Ohtsuki, Y.; Fujimura, Y.; Domcke, W. Optimal control of ultrafast cis-trans photoisomerization of retinal in rhodopsin via a conical intersection. The Journal of chemical physics 2005, 123, 144508.

(54) Flores, S. C.; Batista, V. S. Model study of Coherent-Control of the Femtosecond Primary Event of Vision. J. Phys. Chem. B 2004, 108, 6745-6749.

(55) Tscherbul, T. V.; Brumer, P. Excitation of biomolecules with incoherent light: Quantum yield for the photoisomerization of model retinal. J. Phys. Chem. A 2014, 118, 31003111.

(56) Hahn, S.; Stock, G. Quantum-mechanical modeling of the femtosecond isomerization in rhodopsin. The Journal of Physical Chemistry B 2000, 104, 1146-1149.

(57) Humeniuk, A.; Mitrić, R. Non-adiabatic dynamics around a conical intersection with surface-hopping coupled coherent states. J. Chem. Phys. 2016, 144, 234108.

(58) Lauvergnat, D.; Nauts, A. Exact numerical computation of a kinetic energy operator in curvilinear coordinates. J. Chem. Phys. 2002, 116, 8560-8570.

(59) Nauts, A.; Chapuisat, X. Momentum, quasi-momentum and hamiltonian operators in terms of arbitrary curvilinear coordinates, with special emphasis on molecular hamiltonians. Mol. Phys. 1985, 55, 1287-1318.

(60) Nauts, A.; Lauvergnat, D. Numerical on-the-fly implementation of the action of the kinetic energy operator on a vibrational wave function: application to methanol. Mol. Phys. 2018, 116, 3701-3709.

(61) Ryabinkin, I. G.; Joubert-Doriol, L.; Izmaylov, A. F. When do we need to account for the geometric phase in excited state dynamics? J. Chem. Phys. 2014, 140, 214116. 
(62) Gherib, R.; Ryabinkin, I. G.; Izmaylov, A. F. Why Do Mixed Quantum-Classical Methods Describe Short-Time Dynamics through Conical Intersections So Well? Analysis of Geometric Phase Effects. J. Chem. Theory Comput. 2015, 11, 1375-1382.

(63) Ryabinkin, I. G.; Joubert-Doriol, L.; Izmaylov, A. F. Geometric phase effects in nonadiabatic dynamics near conical intersections. Acc. Chem. Res. 2017, 50, 1785-1793.

(64) Kohen, D.; Stillinger, F. H.; Tully, J. C. Model studies of nonadiabatic dynamics. J. Chem. Phys. 1998, 109, 4713.

(65) Habershon, S.; Manolopoulos, D. E. Zero point energy leakage in condensed phase dynamics: An assessment of quantum simulation methods for liquid water. J. Chem. Phys. 2009, 131, 244518.

(66) Brieuc, F.; Bronstein, Y.; Dammak, H.; Depondt, P.; Finocchi, F.; Hayoun, M. ZeroPoint Energy Leakage in Quantum Thermal Bath Molecular Dynamics Simulations. J. Chem. Theory Comput. 2016, 12, 5688-5697.

(67) Agostini, F. An exact-factorization perspective on quantum-classical approaches to excited-state dynamics. Euro. Phys. J. B 2018, 91, 143.

(68) Weingart, O.; Altoe, P.; Stenta, M.; Bottoni, A.; Orlandi, G.; Garavelli, M. Product formation in rhodopsin by fast hydrogen motions. Phys. Chem. Chem. Phys. 2011, 13, 3645-3648.

(69) Dodina, A.; Brumer, P. Light-induced processes in nature: Coherences in the establishment of the nonequilibrium steady state in model retinal isomerization. J. Chem. Phys. 2019, 150, 184304.

(70) Axelrod, S.; Brumer, P. Multiple time scale open systems: Reaction rates and quantum coherence in model retinal photoisomerization under incoherent excitation. J. Chem. Phys. 2019, 151, 014104. 
(71) Brumer, P. Shedding (incoherent) light on quantum effects in light-induced biological processes. J. Phys. Chem. Lett. 2018, 9, 2946-2955.

(72) Barbatti, M. Simulation of excitation by sunlight in mixed quantum-classical dynamics. J. Chem. Theory Comput. 2020, doi.org/10.1021/acs.jctc.0c00501.

(73) Lauvergnat, D. 2018; QuantumModelLib.

(74) Podolsky, B. Quantum-mechanically correct form of Hamiltonian function for conservative systems. Phys. Rev. 1928, 32, 812-816.

(75) Wilson, E. B.; Decius, J. C.; Cross, P. C.; Sundheim, B. R. Molecular vibrations: The theory of infrared and Raman vibrational spectra, McGraw-Hil ed.; 1955; p 288.

(76) Fábri, C.; Mátyus, E.; Császár, A. G. Numerically constructed internal-coordinate Hamiltonian with Eckart embedding and its application for the inversion tunneling of ammonia. Spectrochimica Acta - Part A: Molecular and Biomolecular Spectroscopy 2014, 119, 84-89.

(77) Nauts, A.; Chapuisat, X. Hamiltonians for constrained N-particle systems. Chem. Phys. Lett. 1987, 136, 164.

(78) Yachmenev, A.; Yurchenko, S. N. Automatic differentiation method for numerical construction of the rotational-vibrational Hamiltonian as a power series in the curvilinear internal coordinates using the Eckart frame. J. Chem. Phys. 2015, 143, 014105.

(79) Tal-Ezer, H.; Kosloff, R. An accurate and efficient scheme for propagating the time dependent Schrödinger equation. J. Chem. Phys. 1984, 81, 3967-3971.

(80) Feynman, R. P. Forces in molecules. Phys. Rev. 1939, 56, 340.

(81) Lengsfield III, B. H.; Yarkony, D. R. Adv. Chem. Phys.; John Wiley \& Sons, Ltd, 2007; pp $1-71$. 
(82) Worth, G.; Cederbaum, L. Beyond Born-Oppenheimer: molecular dynamics through a conical intersection. Annu. Rev. Phys. Chem. 2004, 55, 127-158.

(83) Agostini, F.; Min, S. K.; Tavernelli, I.; Gossel, G. H. 2018; CTMQC. 
Graphical TOC Entry

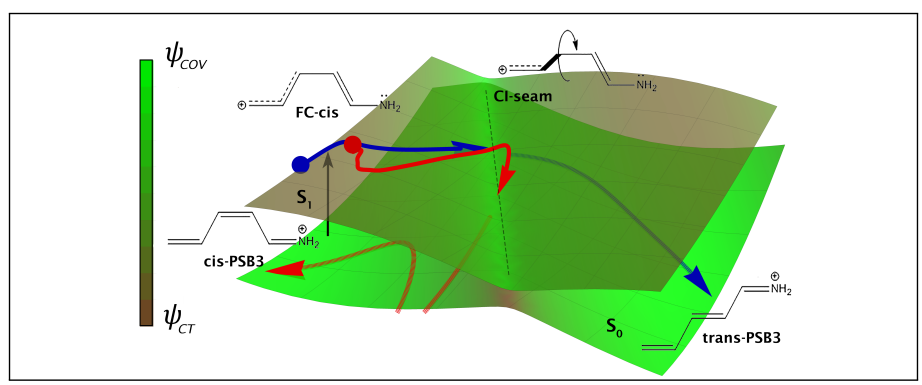

